\title{
Linear-Quadratic Gaussian Balancing for Model Reduction of Differential-Algebraic Systems
}

Jens Möckel Timo Reis Tatjana Stykel

Preprint 2011/01

Preprint-Reihe des Instituts für Mathematik

Technische Universität Berlin

http://www.math.tu-berlin.de/preprints

Preprint 2011/01

January 2011 



\title{
RESEARCH ARTICLE
}

\section{Linear-Quadratic Gaussian Balancing for Model Reduction of Differential-Algebraic Systems}

\author{
Jens Möckel ${ }^{a}$, Timo Reis ${ }^{b *}$ and Tatjana Stykel $^{a}$ \\ ${ }^{a}$ Institut für Mathematik, Technische Universität Berlin, \\ Straße des 17. Juni 136, 10623 Berlin, Germany \\ ${ }^{b}$ Institut für Numerische Simulation, Technische Universität Hamburg-Harburg, \\ Schwarzenbergstraße 95 E, 21073 Hamburg, Germany \\ (February 8, 2011)
}

\begin{abstract}
We generalize the model reduction method of linear-quadratic Gaussian balanced truncation to linear timeinvariant systems governed by differential-algebraic equations. The presented method relies on the solution of generalized algebraic Riccati equations. A simple a priori error bound in the gap metric is given.
\end{abstract}

Keywords: model reduction; descriptor systems; balanced truncation; generalized algebraic Riccati equations; gap metric; normalized coprime factorization;

\section{Introduction}

We consider model order reduction for linear time-invariant continuous-time differential-algebraic systems or, also called, descriptor systems, of the form

$$
\begin{aligned}
E \dot{x}(t) & =A x(t)+B u(t), \\
y(t) & =C x(t)+D u(t),
\end{aligned}
$$

where $E, A \in \mathbb{R}^{n, n}, B \in \mathbb{R}^{n, m}, C \in \mathbb{R}^{p, n}$ and $D \in \mathbb{R}^{p, m}$. Here, $u, x$ and $y$ denote, respectively, the input, state and output of the system, and $x_{0} \in \mathbb{R}^{n}$ is the initial value. We call $n$ the order of system (1). The aim of model reduction is the approximation of (1) by

$$
\begin{aligned}
\widetilde{E} \dot{\tilde{x}}(t) & =\widetilde{A} \widetilde{x}(t)+\widetilde{B} \widetilde{u}(t), \\
\widetilde{y}(t) & =\widetilde{C} \widetilde{x}(t)+\widetilde{D} \widetilde{u}(t)
\end{aligned}
$$

of order $\ell \ll n$. A typical requirement for the reduced-order model (2) is that it almost recapitulates the input-output behavior of the original model (1).

For standard state space systems with $E=I$, a popular family of model reduction methods are those related to balancing and truncation, see, e.g., (Gugercin and Antoulas 2004, Moore 1981). These methods are based on the consideration of two energy functionals: one for the required supply, i.e., the minimal energy that has to be put into the system to steer to a given state vector $x$, and the other one for the available storage, i.e., the maximal energy that can be extracted from 
the system initialized by $x$. Those states $x$ with large required supply and small available storage do not contribute significantly to the input-output behavior of the system and can, therefore, be eliminated without considerably changing the transition behavior of the system. An important property of balancing-related model reduction methods is that they provide computable a priori error bounds.

In Lyapunov-based balanced truncation (Moore 1981), available storage and required supply are determined via Lyapunov matrix equations. This method can be applied to asymptotically stable systems only, and an $\mathcal{H}_{\infty}$-norm error bound in terms of Hankel singular values is provided. This allows to determine the $\mathcal{L}_{2}$-distance between the outputs $y$ and $\widetilde{y}$ of systems (1) and (2) driven by a square integrable input $u=\widetilde{u}$.

Another balancing-related model reduction method is linear-quadratic Gaussian (LQG) balanced truncation (Jonckheere and Silverman 1983). Available storage and required supply are defined via integrals over sum of squares of inputs and outputs, and they can be determined by solving certain algebraic Riccati equations. This method is not restricted to asymptotically stable systems anymore, and it provides error bounds in the gap metric which is, roughly speaking, a measure for the distance of the dynamics of two systems. By the results in (Georgiou and Smith 1990), the availability of a priori error bounds in the gap metric makes LQG balancing perfectly suitable as a model reduction method for unstable plants, and can, therefore, be used for the design of low-order controllers (Curtain 2003).

Lyapunov-based balanced truncation has been generalized to descriptor systems in (Stykel 2004). This method requires the solution of generalized Lyapunov equations which involve socalled spectral projectors that decouple the system into differential and algebraic components. A drawback of this approach is that numerical computation of such projectors may be illconditioned and is, in general, impossible without further knowledge of the structural properties of the system.

In this paper, we aim to extend LQG balanced truncation to descriptor systems. The method we introduce is not based on a decoupling of differential and algebraic parts but rather considers descriptor systems as a whole. It is based on the generalized algebraic Riccati equations of type

$$
\begin{aligned}
A X^{T}+X A^{T}+B B^{T}-\left(X C^{T}+B D^{T}\right)\left(I+D D^{T}\right)^{-1}\left(C X^{T}+D B^{T}\right) & =0 \\
E X^{T}-X E^{T} & =0,
\end{aligned}
$$

and

$$
\begin{array}{r}
A^{T} Y+Y^{T} A+C^{T} C-\left(Y^{T} B+C^{T} D\right)\left(I+D^{T} D\right)^{-1}\left(B^{T} Y+D^{T} C\right)=0 \\
E^{T} Y-Y^{T} E=0 .
\end{array}
$$

This kind of equations has been considered previously in (Kawamoto et al. 1999, Xin 2008, Zhang et al. 2009, Yang et al. 2001) in the context of linear-quadratic optimal control of descriptor systems. The problem with those results on solvability of (3) and (4) is that they impose the too strong requirement of impulse-freeness of the system (Yang et al. 2001), or they are based on rather abstract theoretical conditions on the involved matrices (Kawamoto et al. 1999, Xin 2008, Zhang et al. 2009), which are, in general, difficult to verify. Besides developing an LQG balanced truncation model reduction method for descriptor systems, we also present novel solvability criteria for the generalized Riccati equations (3) and (4) in terms of systems theoretic properties of descriptor systems, such as strong stabilizability and strong detectability.

This paper is organized as follows. Section 2 introduces the notation and some background material from linear algebra and systems theory. In Section 3, we analyze the generalized algebraic Riccati equations (3) and (4) and give criteria for the existence of stabilizing solutions of these equations. Section 4 deals with LQG balanced truncation model reduction for descriptor systems. An error bound in the gap metric is also given. Finally, in Section 5, we present some numerical examples. 


\section{Matrix and Control Theoretic Preliminaries}

Throughout this paper, $\mathbb{R}^{n, m}$ denotes the set of $n \times m$ real matrices, whereas $\mathrm{Gl}_{n}(\mathbb{R})$ stands for the set of $n \times n$ real invertible matrices. We denote by $\mathbb{R}(s)$ the field of real rational functions, and by $\mathbb{R}(s)^{n, m}$ the set of $n \times m$ matrices whose entries belong to $\mathbb{R}(s)$. The set of complex numbers with negative real part is denoted by $\mathbb{C}^{-}$. We denote by $A^{T}$ and $A^{*}$, respectively, the transpose and the conjugate transpose of a matrix $A$. The rank, the image and the kernel of $A$ are denoted by $\operatorname{rank} A, \operatorname{im} A$ and $\operatorname{ker} A$, respectively. Furthermore, $\sigma(A)$ stands for the spectrum of a square matrix $A$. Within block matrices, the symbol $\star$ represents unspecified blocks. The identity of size $n$ is denoted by $I_{n}$ or simply by $I$, if the dimension can be obtained from the context. We use $A>0(A \geq 0)$, if the matrix $A$ is symmetric, positive definite (semi-definite), and $A^{1 / 2}$ stands for a matrix square root of $A \geq 0$. Finally, $\|\cdot\|$ denotes the Euclidean vector norm or the spectral matrix norm. The latter is defined as a maximal singular value of a matrix.

Let $s E-A$ be a matrix pencil with $E, A \in \mathbb{R}^{n, n}$. Then $s E-A$ is called regular if $\operatorname{det}(s E-A) \not \equiv 0$. A pencil $P(s)=s E-A$ is called even if $P(-s)^{T}=P(s)$, i.e., if $E=-E^{T}$ and $A=A^{T}$.

For a regular pencil $s E-A$, finite eigenvalues are the numbers $\lambda \in \mathbb{C} \operatorname{such}$ that $\operatorname{det}(\lambda E-A)=0$. If $E$ is singular, then $s E-A$ has also an eigenvalue at infinity. A pencil $s E-A$ is called stable if all its finite eigenvalues belong to $\mathbb{C}^{-}$. Any regular pencil $s E-A$ can be transformed into the Weierstraß canonical form

$$
W E T=\left[\begin{array}{cc}
I_{n_{f}} & 0 \\
0 & N
\end{array}\right], \quad W A T=\left[\begin{array}{cc}
J & 0 \\
0 & I_{n_{\infty}}
\end{array}\right]
$$

where $W, T \in \mathrm{Gl}_{n}(\mathbb{R}), J \in \mathbb{R}^{n_{f}, n_{f}}$ is in Jordan form and $N \in \mathbb{R}^{n_{\infty}, n_{\infty}}$ is nilpotent (Gantmacher 1959). The eigenvalues of $J$ are the finite eigenvalues of $s E-A$, and $N$ corresponds to the eigenvalue at infinity. The index of nilpotency of $N$ is called the index of $s E-A$. In case of the index of $s E-A$ being not greater than one, we speak of impulse-freeness.

Definition 2.1: $\quad$ A subspace $\mathcal{V} \subset \mathbb{R}^{n}$ is called right deflating subspace of a pencil $s E-A$ with $E, A \in \mathbb{R}^{m, n}$ if for a full column rank matrix $V \in \mathbb{R}^{n, k}$ with $\operatorname{im} V=\mathcal{V}$, there exists an $l \leq k$ and matrices $U \in \mathbb{R}^{m, l}$ and $\hat{E}, \hat{A} \in \mathbb{R}^{l, k}$ such that $E V=U \hat{E}$ and $A V=U \hat{A}$, or, equivalently, $(s E-A) V=U(s \hat{E}-\hat{A})$.

For system (1) with $B \in \mathbb{R}^{n, m}, C \in \mathbb{R}^{p, n}, D \in \mathbb{R}^{p, m}$ and $E, A \in \mathbb{R}^{n, n}$ such that the pencil $s E-A$ is regular, a transfer function $\mathbf{G} \in \mathbb{R}(s)^{p, m}$ is given by

$$
\mathbf{G}(s)=C(s E-A)^{-1} B+D .
$$

Conversely, we refer to (1) as a realization of $\mathbf{G}$ and denote it also by $[E, A, B, C, D]$. The symbol $\Sigma_{n, m, p}$ stands for the set of systems with $n$-dimensional state, $m$-dimensional input and $p$-dimensional output, and we write $[E, A, B, C, D] \in \Sigma_{n, m, p}$.

A transfer function $\mathbf{G} \in \mathbb{R}(s)^{p, m}$ is called proper if $\lim _{s \rightarrow \infty} \mathbf{G}(s)$ exists. If, moreover, $\lim _{s \rightarrow \infty} \mathbf{G}(s)=0$, then $\mathbf{G}$ is called strictly proper. A transfer function $\mathbf{G} \in \mathbb{R}(s)^{p, m}$ is called stable, if all its poles are in $\mathbb{C}^{-}$. To expedite calculations with transfer functions, we also use the notation

$$
\mathbf{G}(s)=\left[\begin{array}{c|c}
s E-A & B \\
\hline C & D
\end{array}\right]
$$

We now recall the concept of system equivalence (Dai 1989, p. 10).

Definition 2.2: Two systems $\left[E_{i}, A_{i}, B_{i}, C_{i}, D_{i}\right] \in \Sigma_{n, m, p}, i=1,2$, are called system equivalent 
if there exist $W, T \in \mathrm{Gl}_{n}(\mathbb{R})$ such that

$$
\left[\begin{array}{cc}
s E_{1}-A_{1} & B_{1} \\
C_{1} & D_{1}
\end{array}\right]=\left[\begin{array}{cc}
W & 0 \\
0 & I_{p}
\end{array}\right]\left[\begin{array}{cc}
s E_{2}-A_{2} & B_{2} \\
C_{2} & D_{2}
\end{array}\right]\left[\begin{array}{cc}
T & 0 \\
0 & I_{m}
\end{array}\right]
$$

It is easy to see that system equivalence is an equivalence relation on $\Sigma_{n, m, p}$ and that two equivalent systems have the same transfer function.

Using the transfer function, certain norms and distance measures for linear control systems can be introduced. The space $\mathcal{R} \mathcal{H}_{2}^{m}$ consists of all strictly proper and stable functions in $\mathbb{R}(s)^{m}$. For $\mathbf{g} \in \mathcal{R H}_{2}^{m}$, the $\mathcal{H}_{2}$-norm is given by

$$
\|\mathbf{g}\|_{2}=\sqrt{\frac{1}{2 \pi} \int_{-\infty}^{\infty}\|\mathbf{g}(i \omega)\|^{2} d \omega}
$$

It is well-known (Zhou et al. 1996, p. 97) that the $\mathcal{H}_{2}$-norm induces an inner product and, via inverse Laplace transform, the space $\mathcal{R H}_{2}^{m}$ can be isometrically and densely embedded in the Lebesgue space $\mathcal{L}_{2}^{m}\left(\mathbb{R}^{+}\right)$of square integrable $\mathbb{R}^{m}$-valued functions defined on $\mathbb{R}^{+}=[0, \infty)$.

The space $\mathcal{H}_{\infty}^{p, m}$ consists of all analytical and bounded $p \times m$-valued functions. We further define $\mathcal{R} \mathcal{H}_{\infty}^{p, m}=\mathcal{H}_{\infty}^{p, m} \cap \mathbb{R}(s)^{p, m}$, i.e., $\mathcal{R} \mathcal{H}_{\infty}^{p, m}$ consists of all stable and proper functions in $\mathbb{R}(s)^{p, m}$. The $\mathcal{H}_{\infty}$-norm of $\mathbf{G} \in \mathcal{R} \mathcal{H}_{\infty}^{p, m}$ is defined by

$$
\|\mathbf{G}\|_{\infty}=\sup _{\omega \in \mathbb{R}}\|\mathbf{G}(i \omega)\|
$$

A (right) coprime factorization of $\mathbf{G} \in \mathbb{R}(s)^{p, m}$ is given by a function $\left[\begin{array}{l}\mathbf{M} \\ \mathbf{N}\end{array}\right]$ with $\mathbf{N} \in \mathcal{R} \mathcal{H}_{\infty}^{p, m}$ and $\mathbf{M} \in \mathcal{R} \mathcal{H}_{\infty}^{m, m}$ such that $\mathbf{G}(s)=\mathbf{N}(s) \mathbf{M}^{-1}(s)$, and there exist $\mathbf{X}_{r} \in \mathcal{R} \mathcal{H}_{\infty}^{m, m}$ and $\mathbf{Y}_{r} \in \mathcal{R} \mathcal{H}_{\infty}^{m, p}$ that satisfy the Bézout identity

$$
\mathbf{X}_{r}(s) \mathbf{M}(s)+\mathbf{Y}_{r}(s) \mathbf{N}(s)=I_{m}
$$

A coprime factorization is called normalized, if, additionally, the equation

$$
\mathbf{M}^{T}(-s) \mathbf{M}(s)+\mathbf{N}^{T}(-s) \mathbf{N}(s)=I_{m}
$$

holds true. The range

$$
\operatorname{im}\left[\begin{array}{l}
\mathbf{M} \\
\mathbf{N}
\end{array}\right]=\left\{\left[\begin{array}{l}
\mathbf{M g} \\
\mathbf{N g}
\end{array}\right]: \mathbf{g} \in \mathcal{R H}_{2}^{m}\right\} \subset \mathcal{H}_{2}^{m+p}
$$

of a normalized coprime factorization expresses the graph of $\mathbf{G}$, i.e., the frequency domain counterpart of the set of input-output pairs of system (1) that realizes $\mathbf{G}$.

Using normalized coprime factorizations, another distance measure for general transfer functions can be introduced.

Definition 2.3: Let transfer functions $\mathbf{G}_{1}, \mathbf{G}_{2} \in \mathbb{R}(s)^{p, m}$ with normalized coprime factorizations $\left[\mathbf{M}_{1}^{T}, \mathbf{N}_{1}^{T}\right]^{T}$ and $\left[\mathbf{M}_{2}^{T}, \mathbf{N}_{2}^{T}\right]^{T}$ be given. Let $\Pi_{1}, \Pi_{2}: \mathcal{R H}_{2}^{m+p} \rightarrow \mathcal{R H}_{2}^{m+p}$ be orthogonal projectors such that

$$
\operatorname{im} \Pi_{1}=\operatorname{im}\left[\begin{array}{l}
\mathbf{M}_{1} \\
\mathbf{N}_{1}
\end{array}\right], \quad \operatorname{im} \Pi_{2}=\operatorname{im}\left[\begin{array}{l}
\mathbf{M}_{2} \\
\mathbf{N}_{2}
\end{array}\right]
$$


Then the gap between $\mathbf{G}_{1}$ and $\mathbf{G}_{2}$ is defined via

$$
\delta_{g}\left(\mathbf{G}_{1}, \mathbf{G}_{2}\right)=\left\|\Pi_{1}-\Pi_{2}\right\|_{L\left(\mathcal{R} \mathcal{H}_{2}^{m+p}\right)},
$$

where $\|\cdot\|_{L\left(\mathcal{R H}_{2}^{m+p}\right)}$ denotes the norm of bounded linear operators mapping $\mathcal{R H}_{2}^{m+p}$ into $\mathcal{R} \mathcal{H}_{2}^{m+p}$.

It is shown in (Vidyasagar 2001) that $\delta_{g}(\cdot, \cdot)$ fulfills the axioms of a metric. The gap metric can alternatively be characterized as follows.

Theorem 2.4: (Sefton and Ober 1993) Let transfer functions $\mathbf{G}_{1}, \mathbf{G}_{2} \in \mathbb{R}(s)^{p, m}$ with normalized coprime factorizations $\left[\mathbf{M}_{1}^{T}, \mathbf{N}_{1}^{T}\right]^{T}$ and $\left[\mathbf{M}_{2}^{T}, \mathbf{N}_{2}^{T}\right]^{T}$ be given. Then

$$
\delta_{g}\left(\mathbf{G}_{1}, \mathbf{G}_{2}\right)=\max \left\{\vec{\delta}_{g}\left(\mathbf{G}_{1}, \mathbf{G}_{2}\right), \vec{\delta}_{g}\left(\mathbf{G}_{2}, \mathbf{G}_{1}\right)\right\}
$$

where $\vec{\delta}_{g}\left(\mathbf{G}_{1}, \mathbf{G}_{2}\right)$ is the directed gap between $\mathbf{G}_{1}$ and $\mathbf{G}_{2}$ that is given by

$$
\vec{\delta}_{g}\left(\mathbf{G}_{1}, \mathbf{G}_{2}\right)=\inf _{\mathbf{Q} \in \mathcal{H}_{\infty}^{m, m}}\left\|\left[\begin{array}{l}
\mathbf{M}_{1} \\
\mathbf{N}_{1}
\end{array}\right]-\left[\begin{array}{l}
\mathbf{M}_{2} \\
\mathbf{N}_{2}
\end{array}\right] \mathbf{Q}\right\|_{\infty}
$$

Similarly to standard state space systems (Lancaster and Rodman 1995), certain system theoretic conditions such stabilizability and detectability will be needed to guarantee the solvability of generalized algebraic Riccati equations. For descriptor systems, these concepts are defined as follows, see (Bunse-Gerstner et al. 1999, Dai 1989). We will present the definitions purely in terms of linear algebraic properties of the matrices involved in (1). For a systems theoretic interpretation of all these concepts, see (Zhou et al. 1996, Sec. 2 \& 3)

Definition 2.5: Let $E, A \in \mathbb{R}^{n, n}, B \in \mathbb{R}^{n, m}$ and $C \in \mathbb{R}^{p, n}$. Let $r=\operatorname{rank} E$ and $Z_{l}, Z_{r} \in \mathbb{R}^{n, n-r}$ be full rank matrices such that im $Z_{l}=\operatorname{ker} E^{T}$ and $\operatorname{im} Z_{r}=\operatorname{ker} E$.

i) The triple $(E, A, B)$ is called finite dynamics stabilizable, if $\operatorname{rank}[\lambda E-A, B]=n$ for all $\lambda \in \mathbb{C} \backslash \mathbb{C}^{-}$.

ii) The triple $(E, A, B)$ is called impulse controllable, if $\operatorname{rank}\left[E, A Z_{r}, B\right]=n$.

iii) The triple $(E, A, B)$ is called strongly stabilizable, if it is both finite dynamics stabilizable and impulse controllable.

iv) The triple $(E, A, C)$ is called finite dynamics detectable, if $\operatorname{rank}\left[\lambda E^{T}-A^{T}, C^{T}\right]=n$ for all $\lambda \in \mathbb{C} \backslash \mathbb{C}^{-}$.

v) The triple $(E, A, C)$ is called impulse observable, if $\operatorname{rank}\left[E^{T}, A^{T} Z_{l}, C^{T}\right]=n$.

vi) The triple $(E, A, C)$ is called strongly detectable, if it is both finite dynamics detectable and impulse observable.

We further call the realization $[E, A, B, C, D]$ finite dynamics stabilizable (detectable), impulse controllable (observable), strongly stabilizable (detectable), if the respective property is fulfilled by the triple $(E, A, B)((E, A, C))$.

\section{Generalized Algebraic Riccati Equations}

In this section, we discuss the solvability and solution structure of the generalized algebraic Riccati equations (3) and (4). For reasons of duality, we focus on equation (4) in most cases. As for standard Riccati equations (Lancaster and Rodman 1995), the set of solutions of generalized algebraic Riccati equations is by far not unique. Before we specify solutions of particular interest, we introduce the matrices

$$
R_{X}=I+D D^{T}, \quad R_{Y}=I+D^{T} D .
$$


It is not difficult to see that $R_{X}>0$ and $R_{Y}>0$. Furthermore, it follows from $D^{T} R_{X}^{-1}=R_{Y}^{-1} D^{T}$ that

$$
I-D R_{Y}^{-1} D^{T}=R_{X}^{-1}, \quad I-D^{T} R_{X}^{-1} D=R_{Y}^{-1} .
$$

Thus, we may rewrite the generalized algebraic Riccati equations (3) and (4) as

$$
\begin{aligned}
\left(A-B D^{T} R_{X}^{-1} C\right) X^{T}+X\left(A-B D^{T} R_{X}^{-1} C\right)^{T}+B R_{Y}^{-1} B^{T}-X C^{T} R_{X}^{-1} C X^{T} & =0, \\
E X^{T}-X E^{T} & =0,
\end{aligned}
$$

and

$$
\begin{aligned}
\left(A-B R_{Y}^{-1} D^{T} C\right)^{T} Y+Y^{T}\left(A-B R_{Y}^{-1} D^{T} C\right)+C^{T} R_{X}^{-1} C-Y^{T} B R_{Y}^{-1} B^{T} Y & =0 \\
E^{T} Y-Y^{T} E & =0
\end{aligned}
$$

respectively.

Definition 3.1: Solutions $X, Y \in \mathbb{R}^{n, n}$ of the generalized algebraic Riccati equations (3) and (4) are called stabilizing, if the pencils

$$
\begin{aligned}
& s E-\left(A-\left(X C^{T}+B D^{T}\right) R_{X}^{-1} C\right), \\
& s E-\left(A-B R_{Y}^{-1}\left(B^{T} Y+D^{T} C\right)\right)
\end{aligned}
$$

are both stable and impulse-free.

Note that equations in (4) are slightly more special than the equations

$$
A^{T} Y+Y^{T} A+Q+Y^{T} R Y=0, \quad E^{T} Y-Y E^{T}=0
$$

with symmetric matrices $Q, R \in \mathbb{R}^{n, n}$ considered in (Kawamoto et al. 1999, Xin 2008, Zhang et al. 2009). There solvability conditions were presented, which are based on the inertia of certain matrices composed of $A, Q$ and $R$. However, these conditions lack of system theoretic interpretation and are difficult to check in practice. The work (Yang et al. 2001) studies the generalized algebraic Riccati equation (4) with $D=0$ and also additionally presumes the rather strong and needless assumption that the pencil $\lambda E-A$ is impulse-free.

It immediately follows that strong stabilizability and strong detectability are necessary for the existence of stabilizing solutions of (3) and (4). The following theorem includes that sufficiency holds as well.

Theorem 3.2: Let $[E, A, B, C, D] \in \Sigma_{n, m, p}$ be strongly stabilizable and strongly detectable. Then the following holds true:

(i) There exist the stabilizing solutions $X$ and $Y$ of the generalized algebraic Riccati equations (3) and (4), respectively.

(ii) The stabilizing solutions $X_{1}$ and $X_{2}$ of (3) and $Y_{1}$ and $Y_{2}$ of (4) satisfy $E X_{1}^{T}=E X_{2}^{T}$ and $E^{T} Y_{1}=E^{T} Y_{2}$.

(iii) A solution $X$ of (3) is stabilizing if and only if $E X^{T} \geq 0$.

(iv) A solution $Y$ of (4) is stabilizing if and only if $E^{T} Y \geq 0$.

(v) There exist the stabilizing solutions $X$ and $Y$ of (3) and (4), respectively, such that $I+X Y^{T}$ is invertible.

\subsection{Auxiliary Results for Generalized Algebraic Riccati Equations}

First, we prove several auxiliary facts which we require for the proof of Theorem 3.2. 
Lemma 3.3: (Kawamoto et al. 1999) Two stabilizing solutions $Y_{1}$ and $Y_{2}$ of the generalized algebraic Riccati equation (4) satisfy $E^{T} Y_{1}=E^{T} Y_{2}$.

Lemma 3.4: Let $[E, A, B, C, D] \in \Sigma_{n, m, p}$ be strongly stabilizable and strongly detectable. $A$ solution $Y$ of (4) is stabilizing if and only if $E^{T} Y \geq 0$.

Proof Using (5) and (6), we rewrite the generalized algebraic Riccati equation (4) as

$$
\breve{A}^{T} Y+Y^{T} \breve{A}+\breve{C}^{T} \breve{C}=0, \quad \breve{E}^{T} Y=Y^{T} \breve{E},
$$

where $\breve{E}=E, \breve{A}=A-B R_{Y}^{-1}\left(B^{T} Y+D^{T} C\right)$ and $\breve{C}=\left[C^{T} R_{X}^{-1 / 2}, Y^{T} B R_{Y}^{-1 / 2}\right]^{T}$. The strong detectability of $(E, A, C)$ clearly implies the strong detectability of $(E, \breve{A}, \breve{C})$. Then the desired equivalence is now a direct consequence of the results in (Takaba et al. 1994) on generalized Lyapunov equations.

The following lemma establishes a relation for solutions of the generalized Riccati equations for two equivalent systems. The proof is straightforward and, therefore, omitted.

Lemma 3.5: Let a realization $[E, A, B, C, D] \in \Sigma_{n, m, p}$ be given and let $W, T \in \mathrm{Gl}_{n}(\mathbb{R})$. Then $X$ and $Y$ are stabilizing solutions of (3) and (4), respectively, if and only if, for $\hat{E}=W E T$, $\hat{A}=W A T, \hat{B}=W B, \hat{C}=C T$, the matrices

$$
\hat{X}=W X T^{-T} \quad \text { and } \quad \hat{Y}=W^{-T} Y T
$$

are stabilizing solutions of

$$
\begin{array}{r}
\hat{A} \hat{X}^{T}+\hat{X} \hat{A}^{T}+\hat{B} \hat{B}^{T}-\left(\hat{X} \hat{C}^{T}+\hat{B} D^{T}\right)\left(I+D D^{T}\right)^{-1}\left(\hat{C} \hat{X}^{T}+D \hat{B}^{T}\right)=0 \\
\hat{E} \hat{X}^{T}-\hat{X} \hat{E}^{T}=0, \\
\hat{A}^{T} \hat{Y}+\hat{Y}^{T} \hat{A}+\hat{C}^{T} \hat{C}-\left(\hat{Y}^{T} \hat{B}+\hat{C}^{T} D\right)\left(I+D^{T} D\right)^{-1}\left(\hat{B}^{T} \hat{Y}+D^{T} \hat{C}\right)=0, \\
\hat{E}^{T} \hat{Y}-\hat{Y}^{T} \hat{E}=0 .
\end{array}
$$

An essential feature of solutions of standard algebraic Riccati equations is their correspondence to certain eigenspaces of Hamiltonian matrices. This concept is now extended to generalized Riccati equations. The role of eigenspaces of Hamiltonian matrices is now inherited by deflating subspaces of the even matrix pencils

$$
\begin{aligned}
& H_{X}(s)=\left[\begin{array}{cc}
C^{T} R_{X}^{-1} C & -s E^{T}+\left(A-B D^{T} R_{X}^{-1} C\right)^{T} \\
s E+A-B D^{T} R_{X}^{-1} C & -B R_{Y}^{-1} B^{T}
\end{array}\right], \\
& H_{Y}(s)=\left[\begin{array}{cc}
B R_{Y}^{-1} B^{T} & -s E+A-B R_{Y}^{-1} D^{T} C \\
s E^{T}+\left(A-B R_{Y}^{-1} D^{T} C\right)^{T} & -C^{T} R_{X}^{-1} C
\end{array}\right]
\end{aligned}
$$

for the generalized algebraic Riccati equations (3) and (4), respectively. It can be easily verified that (4) is equivalent to

$$
H_{Y}(s)\left[\begin{array}{c}
Y \\
-I
\end{array}\right]=\left[\begin{array}{c}
I \\
Y^{T}
\end{array}\right]\left(s E-A+B R_{Y}^{-1}\left(B^{T} Y+D^{T} C\right)\right)
$$

In the following lemma, the eigenstructure of $H_{Y}(s)$ is analyzed.

Lemma 3.6: Let $[E, A, B, C, D] \in \Sigma_{n, m, p}$ be strongly stabilizable and strongly detectable. Then the pencil $H_{Y}(s)$ is regular, impulse-free and has no finite eigenvalues on the imaginary axis. 
Proof First, we prove that $H_{Y}(i \omega)$ is invertible for all $\omega \in \mathbb{R}$. This will imply that $H_{Y}(i \omega)$ is regular and has no purely imaginary finite eigenvalues. Let $x_{1}, x_{2} \in \mathbb{C}^{n}$ satisfy

$$
\left[\begin{array}{cc}
B R_{Y}^{-1} B^{T} & -i \omega E+A-B R_{Y}^{-1} D^{T} C \\
i \omega E^{T}+\left(A-B R_{Y}^{-1} D^{T} C\right)^{T} & -C^{T} R_{X}^{-1} C
\end{array}\right]\left[\begin{array}{l}
x_{1} \\
x_{2}
\end{array}\right]=0 .
$$

Then

$$
\begin{aligned}
0= & {\left[\begin{array}{r}
x_{1} \\
-x_{2}
\end{array}\right]^{*}\left[\begin{array}{cc}
B R_{Y}^{-1} B^{T} & -i \omega E+A-B R_{Y}^{-1} D^{T} C \\
i \omega E^{T}+\left(A-B R_{Y}^{-1} D^{T} C\right)^{T} & -C^{T} R_{X}^{-1} C
\end{array}\right]\left[\begin{array}{l}
x_{1} \\
x_{2}
\end{array}\right] } \\
= & x_{1}^{*} B R_{Y}^{-1} B^{T} x_{1}+x_{2}^{*} C^{T} R_{X}^{-1} C x_{2} \\
& +x_{1}^{*}\left(-i \omega E+A-B R_{Y}^{-1} D^{T} C\right) x_{2}-x_{2}^{*}\left(i \omega E^{T}+A^{T}-C^{T} D R_{Y}^{-1} B^{T}\right) x_{1} .
\end{aligned}
$$

Since the first two summands are real and nonnegative, and the last two summands are purely imaginary, the positive definiteness of $R_{X}$ and $R_{Y}$ implies that $B^{T} x_{1}=0$ and $C x_{2}=0$. Then (9) gives rise to $(-i \omega E+A) x_{2}=0$ and $\left(i \omega E^{T}+A^{T}\right) x_{1}=0$. Thus, due to finite dynamics stabilizability and finite dynamics detectability of $[E, A, B, C, D]$, we obtain that $x_{1}=0$ and $x_{2}=0$.

It remains to show that the index of $H_{Y}(s)$ is at most one. Assume that $x_{10}, x_{20}, x_{11}, x_{21} \in \mathbb{R}^{n}$ such that

$$
\left[\begin{array}{cc}
0 & -E \\
E^{T} & 0
\end{array}\right]\left[\begin{array}{l}
x_{10} \\
x_{20}
\end{array}\right]=0, \quad\left[\begin{array}{cc}
B R_{Y}^{-1} B^{T} & A-B R_{Y}^{-1} D^{T} C \\
\left(A-B R_{Y}^{-1} D^{T} C\right)^{T} & -C^{T} R_{X}^{-1} C
\end{array}\right]\left[\begin{array}{c}
x_{10} \\
x_{20}
\end{array}\right]=\left[\begin{array}{cc}
0 & -E \\
E^{T} & 0
\end{array}\right]\left[\begin{array}{l}
x_{11} \\
x_{21}
\end{array}\right]
$$

In particular, we have $E^{T} x_{10}=0$ and $E x_{20}=0$. Then a multiplication of the second equation from the left by the row vector $\left[x_{10}^{T},-x_{20}^{T}\right]$ gives

$$
\begin{aligned}
& x_{10}^{T} B R_{Y}^{-1} B^{T} x_{10}+x_{20}^{T} C^{T} R_{X}^{-1} C x_{20} \\
& \quad+x_{10}^{T}\left(A-B R_{Y}^{-1} D^{T} C\right) x_{20}-x_{20}^{T}\left(A-B R_{Y}^{-1} D^{T} C\right)^{T} x_{10} \\
& =-x_{10}^{T} E x_{21}-x_{20}^{T} E^{T} x_{11}=0
\end{aligned}
$$

Applying the same argumentation as before, we obtain that $B^{T} x_{10}=0$ and $C x_{20}=0$. Therefore,

$$
\begin{aligned}
E x_{20} & =0, \quad A x_{20}=-E x_{21}, \quad C x_{20}=0, \\
E^{T} x_{10} & =0, \quad A^{T} x_{10}=E^{T} x_{11}, \quad B^{T} x_{10}=0,
\end{aligned}
$$

or, equivalently,

$$
\left[\begin{array}{c}
E \\
Z_{l}^{T} A \\
C
\end{array}\right] x_{20}=0, \quad\left[\begin{array}{c}
E^{T} \\
Z_{r}^{T} A^{T} \\
B^{T}
\end{array}\right] x_{10}=0
$$

The assumption of impulse observability and impulse controllability then leads to $x_{10}=0$ and $x_{20}=0$.

Lemma 3.7: Let $[E, A, B, C, D] \in \Sigma_{n, m, p}$ be strongly stabilizable and strongly detectable and let $r=\operatorname{rank} E$. Then $H_{Y}(s)$ has a unique $r$-dimensional deflating subspace $\mathcal{V}$ corresponding to the eigenvalues in the open left half-plane. Furthermore, $v^{T} H_{Y}(s) v=0$ for all $v \in \mathcal{V}$.

Proof Since $H_{Y}(s)$ is impulse-free and has no purely imaginary finite eigenvalues, the deflating subspace corresponding to the finite eigenvalues is of dimension $2 r$. The existence of a unique 
$r$-dimensional deflating subspace $\mathcal{V}$ corresponding to the eigenvalues in $\mathbb{C}^{-}$is now a consequence of the results in (Thompson 1976) for even matrix pencils.

Let $V, U \in \mathbb{R}^{2 n, r}$ and $A_{-} \in \mathbb{R}^{r, r}$ with $\operatorname{im} V=\mathcal{V}$ and $\sigma\left(A_{-}\right) \subset \mathbb{C}^{-}$satisfy

$$
H_{Y}(s) V=U\left(s I-A_{-}\right) .
$$

Then for $H_{Y}(s)=s \mathcal{E}-\mathcal{A}$ holds $U=\mathcal{E} V$ and, thus, $\mathcal{E} V A_{-}=\mathcal{A} V$. Using $\mathcal{E}^{T}=-\mathcal{E}$ and $\mathcal{A}^{T}=\mathcal{A}$, we obtain that

$$
V^{T} \mathcal{E} V A_{-}=V^{T} \mathcal{A} V=A_{-}^{T} V^{T} \mathcal{E}^{T} V=-A_{-}^{T} V^{T} \mathcal{E} V
$$

Hence, $V^{T} \mathcal{E} V$ fulfills the Lyapunov equation

$$
A_{-}^{T}\left(V^{T} \mathcal{E} V\right)+\left(V^{T} \mathcal{E} V\right) A_{-}=0
$$

Since $A_{-}$is stable, we get $V^{T} \mathcal{E} V=0$. Hence, $V^{T} \mathcal{A} V=V^{T} \mathcal{E} V A_{-}=0$. Altogether, we have $V^{T} H_{Y}(s) V=0$ or, equivalently, $v^{T} H_{Y}(s) v=0$ for all $v \in \mathcal{V}$.

Lemma 3.8: Let $[E, A, B, C, D] \in \Sigma_{n, m, p}$ strongly stabilizable and strongly detectable and let $r=\operatorname{rank} E$. Let $\mathcal{V}$ be an $r$-dimensional deflating subspace of $H_{Y}(s)$ corresponding to the eigenvalues in $\mathbb{C}^{-}$. Let $V_{1}, V_{2} \in \mathbb{R}^{n, r}$ such that $\mathcal{V}=\operatorname{im}\left[\begin{array}{l}V_{1} \\ V_{2}\end{array}\right]$. Then $\operatorname{rank}\left(E V_{2}\right)=r$.

Proof Let $W_{1}, W_{2} \in \mathbb{R}^{n, r}$ and $A_{-} \in \mathbb{R}^{r, r}$ with $\sigma\left(A_{-}\right) \subset \mathbb{C}^{-}$satisfy

$$
\left[\begin{array}{cc}
B R_{Y}^{-1} B^{T} & -s E+A-B R_{Y}^{-1} D^{T} C \\
s E^{T}+\left(A-B R_{Y}^{-1} D^{T} C\right)^{T} & -C^{T} R_{X}^{-1} C
\end{array}\right]\left[\begin{array}{c}
V_{1} \\
V_{2}
\end{array}\right]=\left[\begin{array}{c}
W_{1} \\
W_{2}
\end{array}\right]\left(s I-A_{-}\right) .
$$

Then

$$
\left[\begin{array}{cc}
0 & -E \\
E^{T} & 0
\end{array}\right]\left[\begin{array}{l}
V_{1} \\
V_{2}
\end{array}\right] A_{-}=\left[\begin{array}{cc}
B R_{Y}^{-1} B^{T} & A-B R_{Y}^{-1} D^{T} C \\
\left(A-B R_{Y}^{-1} D^{T} C\right)^{T} & -C^{T} R_{X}^{-1} C
\end{array}\right]\left[\begin{array}{l}
V_{1} \\
V_{2}
\end{array}\right]
$$

The stability of $A_{-}$clearly implies its invertibility.

Assume now that $\operatorname{rank}\left(E V_{2}\right)<r$. Then we can show that the space $\operatorname{ker}\left(E V_{2}\right)$ is $A_{-}^{-1}$-invariant. For any $v \in \operatorname{ker}\left(E V_{2}\right)$, we find that $w=A_{-}^{-1} v$ fulfills

$$
B R_{Y}^{-1} B^{T} V_{1} w+\left(A-B R_{Y}^{-1} D^{T} C\right) V_{2} w=0 .
$$

Making use of this identity, we obtain that

$$
\begin{aligned}
0 & =\left[\begin{array}{c}
V_{1} w \\
V_{2} w
\end{array}\right]^{T}\left[\begin{array}{cc}
B R_{Y}^{-1} B^{T} & A-B R_{Y}^{-1} D^{T} C \\
\left(A-B R_{Y}^{-1} D^{T} C\right)^{T} & -C^{T} R_{X}^{-1} C
\end{array}\right]\left[\begin{array}{l}
V_{1} w \\
V_{2} w
\end{array}\right] \\
& =w^{T} V_{2}^{T}\left(A-B R_{Y}^{-1} D^{T} C\right)^{T} V_{1} w-w^{T} V_{2}^{T} C^{T} R_{X}^{-1} C V_{2} w \\
& =\left(\left(A-B R_{Y}^{-1} D^{T} C\right) V_{2} w\right)^{T} V_{1} w-w^{T} V_{2}^{T} C^{T} R_{X}^{-1} C V_{2} w \\
& =-w^{T} V_{1}^{T} B R_{Y}^{-1} B^{T} V_{1} w-w^{T} V_{2}^{T} C^{T} R_{X}^{-1} C V_{2} w
\end{aligned}
$$

and, hence, $B^{T} V_{1} w=0$ and $C V_{2} w=0$. Once again using (10), we get $A V_{2} w=0$. The finite dynamics detectability of $(E, A, C)$ particularly implies that $\left[0 \cdot E-A^{T}, C^{T}\right]=\left[-A^{T}, C^{T}\right]$ has full row rank. Hence, $V_{2} A_{-}^{-1} v=V_{2} w=0$. Thus, $\operatorname{ker}\left(E V_{2}\right)$ is $A_{-}^{-1}$-invariant. This implies that $\operatorname{ker}\left(E V_{2}\right)$ is also $A_{-}$-invariant. 
Let the columns of $V_{0} \in \mathbb{R}^{n, r_{0}}, r_{0}>0$, form a basis of $\operatorname{ker}\left(E V_{2}\right)$. Then the columns of the matrix

$$
\left[\begin{array}{l}
V_{10} \\
V_{20}
\end{array}\right]=\left[\begin{array}{l}
V_{1} V_{0} \\
V_{2} V_{0}
\end{array}\right]
$$

span an $r_{0}$-dimensional deflating subspace of $H_{Y}(s)$. Therefore, there exist $A_{0} \in \mathbb{R}^{r_{0}, r_{0}}$ with $\sigma\left(A_{0}\right) \subset \sigma\left(A_{-}\right) \subset \mathbb{C}^{-}$and $W_{10}, W_{20} \in \mathbb{R}^{n, r_{0}}$ such that

$$
\left[\begin{array}{cc}
B R_{Y}^{-1} B^{T} & -s E+A-B R_{Y}^{-1} D^{T} C \\
s E^{T}+\left(A-B R_{Y}^{-1} D^{T} C\right)^{T} & -C^{T} R_{X}^{-1} C
\end{array}\right]\left[\begin{array}{c}
V_{10} \\
V_{20}
\end{array}\right]=\left[\begin{array}{c}
W_{10} \\
W_{20}
\end{array}\right]\left(s I-A_{0}\right) .
$$

By argumenting as before, we obtain that $B^{T} V_{10}=0$ and $C V_{20}=0$. Then the relation

$$
\left[\begin{array}{cc}
0 & -s E+A \\
s E^{T}+A^{T} & 0
\end{array}\right]\left[\begin{array}{l}
V_{10} \\
V_{20}
\end{array}\right]=\left[\begin{array}{l}
W_{10} \\
W_{20}
\end{array}\right]\left(s I-A_{0}\right)
$$

holds true. In particular, for an eigenvector $v_{0} \in \mathbb{C}^{r_{0}} \backslash\{0\}$ of $A_{0}$ corresponding to the eigenvalue $\lambda \in \mathbb{C}^{-}$, we have

$$
\left(-\lambda E^{T}-A^{T}\right) V_{10} v_{0}=-W_{20}\left(\lambda I-A_{0}\right) v_{0}=0 .
$$

Together with $B^{T} V_{10} v_{0}=0$, this implies that $\left(V_{10} v_{0}\right)^{*}[-\bar{\lambda} E-A, B]=0$. Due to the assumption of finite dynamics stabilizability, we have $V_{10} v_{0}=0$. This implies $W_{20} v_{0}=0$. Since, on the other hand, we have $W_{10} v_{0}=-E V_{20} v_{0}=0$, the relation

$$
r_{0}=\operatorname{rank}\left[\begin{array}{c}
V_{10} \\
V_{20}
\end{array}\right]=\operatorname{rank}\left[\begin{array}{c}
W_{10} \\
W_{20}
\end{array}\right]<r_{0}
$$

holds true. This is a contradiction. Thus, $\operatorname{rank}\left(E V_{2}\right)=r$.

We now characterize a set of stabilizing solutions in the case where $E$ has a special structure.

Definition 3.9: We call a realization $[E, A, B, C, D] \in \Sigma_{n, m, p}$ with $r=\operatorname{rank} E$ semi-explicit if

$$
E=\left[\begin{array}{rr}
I_{r} & 0 \\
0 & 0
\end{array}\right], \quad A=\left[\begin{array}{ll}
A_{11} & A_{12} \\
A_{21} & A_{22}
\end{array}\right], \quad B=\left[\begin{array}{l}
B_{1} \\
B_{2}
\end{array}\right], \quad C=\left[\begin{array}{ll}
C_{1} & C_{2}
\end{array}\right]
$$

where $A_{11} \in \mathbb{R}^{r, r}, A_{12} \in \mathbb{R}^{r, n-r}, A_{21} \in \mathbb{R}^{n-r, r}, A_{22} \in \mathbb{R}^{n-r, n-r}, B_{1} \in \mathbb{R}^{r, m}, B_{2} \in \mathbb{R}^{n-r, m}$ and $C_{1} \in \mathbb{R}^{p, r}, C_{2} \in \mathbb{R}^{p, n-r}$.

Note that any realization can be transformed into a semi-explicit form by computing, for example, a singular value decomposition of $E$. The name semi-explicit is due to the property that $E$ structured as in (11) causes that a part of the derivative of the state vector occurs explicitly, while the remaining part is of pure algebraic nature.

Lemma 3.10: Let $[E, A, B, C, D] \in \Sigma_{n, m, p}$ be a strongly stabilizable and strongly detectable realization in the semi-explicit form (11). Let $\mathcal{V}$ be an $r$-dimensional deflating subspace of $H_{Y}(s)$ corresponding to the eigenvalues in $\mathbb{C}^{-}$and let

$$
\mathcal{V}=\operatorname{im}\left[\begin{array}{l}
V_{11} \\
V_{12} \\
V_{21} \\
V_{22}
\end{array}\right]
$$


for some $V_{11}, V_{21} \in \mathbb{R}^{r, r}, V_{12}, V_{22} \in \mathbb{R}^{n-r, r}$. Then $V_{21}$ is invertible. Moreover, $Y \in \mathbb{R}^{n, n}$ is a stabilizing solution of the generalized Riccati equation (4) if and only if

$$
Y=\left[\begin{array}{cc}
V_{11} V_{21}^{-1} & 0 \\
\left(V_{12}-Y_{0} V_{22}\right) V_{21}^{-1} & Y_{0}
\end{array}\right]
$$

where $Y_{0} \in \mathbb{R}^{n-r, n-r}$ solves the quadratic equation

$$
\left(A_{22}-B_{2} R_{Y}^{-1} D^{T} C_{2}\right)^{T} Y_{0}+Y_{0}^{T}\left(A_{22}-B_{2} R_{Y}^{-1} D^{T} C_{2}\right)+C_{2}^{T} R_{X}^{-1} C_{2}-Y_{0}^{T} B_{2} R_{Y}^{-1} B_{2}^{T} Y_{0}=0 .
$$

Proof The invertibility of $V_{21}$ follows from Lemma 3.8. The remaining statements are proven in Lemma 1 of (Kawamoto et al. 1999).

\subsection{Proof of Theorem 3.2}

We are now prepared to prove the main result of this section. For the generalized Riccati equation (4), the statements (ii) and (iv) of Theorem 3.2 immediately follow from Lemmas 3.3 and 3.4. The corresponding results for (3) can be obtained by duality. Since (i) is a direct consequence of $(\mathrm{v})$, it remains to show (v) only.

By Lemma 3.5, we have that for invertible matrices $W, T \in \mathbb{R}^{n, n}$, stabilizing solutions $X$ and $Y$ of the generalized algebraic Riccati equations (3) and (4) are related to those associated with the system $[\hat{E}, \hat{A}, \hat{B}, \hat{C}, D]=[W E T, W A T, W B, C T, D]$ via $\hat{X}=W X T^{-T}, \hat{Y}=W^{-T} Y T$. Consequently, $I+X Y^{T}=W^{-1}\left(I+\hat{X} \hat{Y}^{T}\right) W$ and, hence, it suffices to prove the statement for the semi-explicit case (11) with, additionally, $A_{22}=A_{22}^{T} \leq 0$. Such a form can always be achieved by an appropriate choice of $W$ and $T$.

Let

$$
\mathcal{V}_{X}=\operatorname{im}\left[\begin{array}{l}
V_{11 X} \\
V_{12 X} \\
V_{21 X} \\
V_{22 X}
\end{array}\right], \quad \mathcal{V}_{Y}=\operatorname{im}\left[\begin{array}{l}
V_{11 Y} \\
V_{12 Y} \\
V_{21 Y} \\
V_{22 Y}
\end{array}\right]
$$

with $V_{11 X}, V_{21 X}, V_{11 Y}, V_{21 Y} \in \mathbb{R}^{r, r}, V_{12 X}, V_{22 X}, V_{12 Y}, V_{22 Y} \in \mathbb{R}^{n-r, r}$ be the $r$-dimensional stable deflating subspaces of the pencils $H_{X}(s)$ and $H_{Y}(s)$ as in (7) and (8). Then by Lemma 3.10 stabilizing solutions of (3) and (4) are given by

$$
X=W^{-1}\left[\begin{array}{cc}
V_{11 X} V_{21 X}^{-1} & 0 \\
\left(V_{12 X}-X_{0}^{T} V_{22 X}\right) V_{21 X}^{-1} & X_{0}^{T}
\end{array}\right]^{T} T^{T}, \quad Y=W^{T}\left[\begin{array}{cc}
V_{11 Y} V_{21 Y}^{-1} & 0 \\
\left(V_{12 Y}-Y_{0} V_{22 Y}\right) V_{21 Y}^{-1} & Y_{0}
\end{array}\right] T^{-1},
$$

where $X_{0}, Y_{0} \in \mathbb{R}^{n-r, n-r}$ solve the equations

$$
\begin{aligned}
& \left(A_{22}-B_{2} D^{T} R_{X}^{-1} C_{2}\right) X_{0}^{T}+X_{0}\left(A_{22}-B_{2} D^{T} R_{X}^{-1} C_{2}\right)^{T}+B_{2} R_{Y}^{-1} B_{2}^{T}-X_{0} C_{2}^{T} R_{X}^{-1} C_{2} X_{0}^{T}=0 \\
& \left(A_{22}-B_{2} R_{Y}^{-1} D^{T} C_{2}\right)^{T} Y_{0}+Y_{0}^{T}\left(A_{22}-B_{2} R_{Y}^{-1} D^{T} C_{2}\right)+C_{2}^{T} R_{X}^{-1} C_{2}-Y_{0}^{T} B_{2} R_{Y}^{-1} B_{2}^{T} Y_{0}=0
\end{aligned}
$$

Lemma 3.4 implies that the matrices $-V_{11 X} V_{21 X}^{-1}$ and $-V_{11 Y} V_{21 Y}^{-1}$ are both symmetric and positive semi-definite. Then $I+\left(-V_{11 X} V_{21 X}^{-1}\right)\left(-V_{11 Y} V_{21 Y}^{-1}\right)$ is invertible. Hence, it suffices to show that there exist solutions $X_{0}$ and $Y_{0}$ of (13) and (14) such that $I+X_{0} Y_{0}^{T}$ is invertible. Since $(E, A, B)$ is impulse controllable and $(E, A, C)$ is impulse observable, we have $\operatorname{rank}\left[A_{22}, B_{2}\right]=\operatorname{rank}\left[A_{22}^{T}, C_{2}^{T}\right]=n-r$. Furthermore, since $\lambda=0$ is the only eigenvalue of $A_{22}$ which is not in $\mathbb{C}^{-}$, the triple $\left(I, A_{22}, B_{2}\right)$ is stabilizable and $\left(I, A_{22}, C_{2}\right)$ is detectable. Hence, 
the triples $\left(I, A_{22}-B_{2} R_{Y}^{-1} D^{T} C_{2}, B_{2}\right)$ and $\left(I, A_{22}-B_{2} D^{T} R_{X}^{-1} C_{2}, C_{2}\right)$ are also stabilizable and detectable, respectively. Using the theory for standard algebraic Riccati equations (Lancaster and Rodman 1995), equations (13) and (14) have symmetric solutions such that $X_{0} \geq 0$ and $Y_{0} \geq 0$. As a consequence, the matrix $I+X_{0} Y_{0}^{T}$ is invertible. Hence, this also holds true for $I+X Y^{T}$

\subsection{Lyapunov -based Balanced Truncation for Coprime Factorization}

We now show that the stabilizing solution $Y$ of the generalized Riccati equation (4) can be used to construct a normalized coprime factorization of the realization $[E, A, B, C, D] \in \Sigma_{n, m, p}$ of $\mathbf{G} \in \mathbb{R}(s)^{p, m}$. The subsequent result is proven in (Liu et al. 1997) for the case $D=0$. Since an extension to general $D$ is straightforward, we omit the proof.

Theorem 3.11: Let a realization $[E, A, B, C, D] \in \Sigma_{n, m, p}$ of $\mathbf{G} \in \mathbb{R}(s)^{p, m}$ be strongly stabilizable and strongly detectable, and let $Y$ be a stabilizing solution of the generalized algebraic Riccati equation (4). Then a normalized right coprime factorization of $\mathbf{G}$ is given by

$$
\left[\begin{array}{c}
\mathbf{M}(s) \\
\mathbf{N}(s)
\end{array}\right]=\left[\begin{array}{c|c}
s E-A-B K & B R_{Y}^{-1 / 2} \\
\hline K & R_{Y}^{-1 / 2} \\
C+D K & D R_{Y}^{-1 / 2}
\end{array}\right]=:\left[\begin{array}{c|c}
s E_{\mathrm{rcf}}-A_{\mathrm{rcf}} & B_{\mathrm{rcf}} \\
\hline C_{\mathrm{rcf}} & D_{\mathrm{rcf}}
\end{array}\right]
$$

where $K=-R_{Y}^{-1}\left(B^{T} Y+D^{T} C\right)$.

Next we prove that the stabilizing solutions of the generalized algebraic Riccati equations (3) and (4) solve certain generalized Lyapunov equations associated with the realization (15) of the normalized coprime factorization of $\mathbf{G}$.

Theorem 3.12: $\quad$ Let a realization $[E, A, B, C, D] \in \Sigma_{n, m, p}$ of $\mathbf{G} \in \mathbb{R}(s)^{p, m}$ be strongly stabilizable and strongly detectable, and let $X$ and $Y$ be the stabilizing solutions of the generalized Riccati equations (3) and (4) such that $I+X Y^{T}$ is invertible. Then the matrices $P=\left(I+X Y^{T}\right)^{-1} X$ and $Q=Y$ solve the generalized Lyapunov equations

$$
\begin{array}{ll}
A_{\mathrm{rcf}} P^{T}+P A_{\mathrm{rcf}}^{T}+B_{\mathrm{rcf}} B_{\mathrm{rcf}}^{T}=0, & E_{\mathrm{rcf}} P^{T}-P E_{\mathrm{rcf}}^{T}=0, \\
A_{\mathrm{rcf}}^{T} Q+Q^{T} A_{\mathrm{rcf}}+C_{\mathrm{rcf}}^{T} C_{\mathrm{rcf}}=0, & E_{\mathrm{rcf}}^{T} Q-Q^{T} E_{\mathrm{rcf}}=0,
\end{array}
$$

where $E_{\mathrm{rcf}} A_{\mathrm{rcf}}, B_{\mathrm{rcf}}$ and $C_{\mathrm{rcf}}$ are as in (15).

Proof Equation (17) directly follows from (4) and the definition of $E_{\mathrm{rcf}}, A_{\mathrm{rcf}}, B_{\mathrm{rcf}}$ and $C_{\mathrm{rcf}}$. In order to show (16), we first observe that the relation $E_{\mathrm{rcf}} P^{T}=P E_{\mathrm{rcf}}^{T}$ is an immediate consequence of

$$
E^{T}\left(I+Y X^{T}\right)=\left(I+Y^{T} X\right) E^{T}, \quad\left(I+X Y^{T}\right)^{-1} X=X\left(I+Y^{T} X\right)^{-1} .
$$

For the remaining part, consider the identities

$$
\begin{aligned}
-\left(I+X Y^{T}\right) B_{\mathrm{rcf}} B_{\mathrm{rcf}}^{T}\left(I+Y X^{T}\right)= & -B R_{Y}^{-1} B^{T}-X Y^{T} B R_{Y}^{-1} B^{T}-B R_{Y}^{-1} B^{T} Y X^{T} \\
& -X Y^{T} B R_{Y}^{-1} B^{T} Y X^{T} \\
- & B R_{Y}^{-1} B^{T}=\left(A-B D^{T} R_{X}^{-1} C\right) X^{T}+X\left(A-B D^{T} R_{X}^{-1} C\right)^{T}-X C^{T} R_{X}^{-1} C X^{T} \\
-C^{T} R_{X}^{-1} C=\left(A-B R_{Y}^{-1} D^{T} C\right)^{T} Y & +Y^{T}\left(A-B R_{Y}^{-1} D^{T} C\right)-Y^{T} B R_{Y}^{-1} B^{T} Y
\end{aligned}
$$


Substituting (20) in (19) and (19) in (18), we obtain that

$$
\begin{aligned}
-B_{\mathrm{rcf}} B_{\mathrm{rcf}}^{T}= & \left(I+X Y^{T}\right)^{-1}\left(\left(A-B D^{T} R_{X}^{-1} C\right) X^{T}+X\left(A-B D^{T} R_{X}^{-1} C\right)^{T}\right. \\
& +X Y^{T}\left(A-B R_{Y}^{-1} D^{T} C\right) X^{T}+X\left(A-B R_{Y}^{-1} D^{T} C\right)^{T} Y X^{T} \\
& -X Y^{T} B R_{Y}^{-1} B^{T} Y X^{T}-X Y^{T} B R_{Y}^{-1} B^{T} \\
& \left.-B R_{Y}^{-1} B^{T} Y X^{T}-X Y^{T} B R_{Y}^{-1} B^{T} Y X^{T}\right)\left(I+Y X^{T}\right)^{-1}
\end{aligned}
$$

Reordering the terms in the right-hand side, we have

$$
\begin{aligned}
-B_{\mathrm{rcf}} B_{\mathrm{rcf}}^{T}= & \left(I+X Y^{T}\right)^{-1}\left(\left(I+X Y^{T}\right)\left(A-B R_{Y}^{-1}\left(B^{T} Y+D^{T} C\right)\right) X^{T}\right. \\
& \left.+X\left(A-B R_{Y}^{-1}\left(B^{T} Y+D^{T} C\right)\right)\left(I+Y X^{T}\right)\right)\left(I+Y X^{T}\right)^{-1} \\
= & A_{\mathrm{rcf}} P^{T}+P A_{\mathrm{rcf}}^{T} .
\end{aligned}
$$

Thus, equation (16) is fulfilled.

Theorem 3.12 shows that solutions $P$ and $Q$ of the generalized Lyapunov equations can be constructed from the stabilizing solutions $X$ and $Y$ of the generalized Riccati equations (3) and (4). It is also well known for standard systems, that the solutions can be used for model reduction by balanced truncation. We will now present an accordant result for stable and impulse-free descriptor systems $[E, A, B, C] \in \Sigma_{n, m, p}$ and can be seen as an alternative to (Stykel 2004) for Lyapunov-based balanced truncation of descriptor systems. In the following lemma we consider balanced truncation of semi-explicit realizations. One can show that the solutions of the Lyapunov equations (16) and (17) have the structure

$$
P=\left[\begin{array}{cc}
\Gamma & \star \\
0 & \star
\end{array}\right], \quad Q=\left[\begin{array}{cc}
\Theta & 0 \\
\star & \star
\end{array}\right] .
$$

Similar as in the case of standard systems, we may apply a further state space transformation which preserves semi-explicit structure and, moreover, the blocks $\Gamma$ and $\Theta$ in the transformed Gramians are diagonal.

Lemma 3.13: Let a stable, impulse-free and semi-explicit system $[\breve{E}, \breve{A}, \breve{B}, \breve{C}, \breve{D}] \in \Sigma_{n, m, p}$ be given and let $r=\operatorname{rank} \breve{E}$. Let $P, Q \in \mathbb{R}^{n, n}$ fulfill the corresponding generalized Lyapunov equations

$$
\begin{array}{ll}
\breve{A} P^{T}+P \breve{A}^{T}+\breve{B} \breve{B}^{T}=0, & \breve{E} P^{T}-P \breve{E}^{T}=0, \\
\breve{A}^{T} Q+Q^{T} \breve{A}+\breve{C}^{T} \breve{C}=0, & \breve{E}^{T} Q-Q^{T} \breve{E}=0 .
\end{array}
$$

Further, assume that for some diagonal matrices $\Gamma=\operatorname{diag}\left(\gamma_{1}, \ldots, \gamma_{r}\right) \in \mathbb{R}^{r, r}$ and $\Theta=\operatorname{diag}\left(\vartheta_{1}, \ldots, \vartheta_{r}\right) \in \mathbb{R}^{r, r}$ with non-negative numbers $\gamma_{1}, \ldots, \gamma_{r}, \vartheta_{1}, \ldots, \vartheta_{r}$ such that

$$
\gamma_{1} \vartheta_{1} \geq \gamma_{2} \vartheta_{2} \geq \ldots \geq \gamma_{\ell} \vartheta_{\ell}>\gamma_{\ell+1} \vartheta_{\ell+1} \geq \ldots \geq \gamma_{r} \vartheta_{r}
$$

there holds

$$
P=\left[\begin{array}{cc}
\Gamma & \star \\
0 & \star
\end{array}\right], \quad Q=\left[\begin{array}{cc}
\Theta & 0 \\
\star & \star
\end{array}\right]
$$


Then for

$$
S_{\ell}=\left[\begin{array}{ccc}
I_{\ell} & 0 & 0 \\
0 & 0 & I_{n-r}
\end{array}\right]
$$

and the matrices $\widetilde{E}=S_{\ell} \breve{E} S_{\ell}^{T}, \widetilde{A}=S_{\ell} \breve{A} S_{\ell}^{T}, \widetilde{B}=S_{\ell} \breve{B}, \widetilde{C}=\breve{C} S_{\ell}^{T} \widetilde{P}=S_{\ell} P S_{\ell}^{T}, \widetilde{Q}=S_{\ell} Q S_{\ell}^{T}$ holds

$$
\begin{array}{ll}
\widetilde{A} \widetilde{P}^{T}+\widetilde{P} \widetilde{A}^{T}+\widetilde{B} \widetilde{B}^{T}=0, & \widetilde{E} \widetilde{P}^{T}-\widetilde{P} \widetilde{E}^{T}=0, \\
\widetilde{A}^{T} \widetilde{Q}+\widetilde{Q}^{T} \widetilde{A}+\widetilde{C}^{T} \widetilde{C}=0, & \widetilde{E}^{T} \widetilde{Q}-\widetilde{Q}^{T} \widetilde{E}=0 .
\end{array}
$$

Moreover, the transfer function

$$
\widetilde{\mathbf{G}}(s)=\left[\begin{array}{c|c|c}
s \widetilde{E}-\widetilde{A} & \widetilde{B} \\
\hline \widetilde{C} & D
\end{array}\right]
$$

fulfills

$$
\|\mathbf{G}-\widetilde{\mathbf{G}}\|_{\infty} \leq 2 \sum_{k=\ell+1}^{r} \sqrt{\gamma_{k} \vartheta_{k}} .
$$

Proof The validity of (22) follows by simple calculations. To show (24), we first make use of (Takaba et al. 1994) to see that the solvability of (21) implies that $s E-A$ is impulse-free. As a consequence, we have that for the partition as in (11) the matrix $A_{22} \in \mathbb{R}^{n-r, n-r}$ is invertible and $\mathbf{G}$ can be represented as

$$
\mathbf{G}(s)=\left(C_{1}-C_{2} A_{22}^{-1} A_{21}\right)\left(s I_{r}-\left(A_{11}-A_{12} A_{22}^{-1} A_{21}\right)\right)^{-1}\left(B_{1}-A_{12} A_{22}^{-1} B_{2}\right) .
$$

Analogously, partitioning system (23) as

$$
\widetilde{E}=\left[\begin{array}{cc}
I_{\ell} & 0 \\
0 & 0
\end{array}\right], \quad \widetilde{A}=\left[\begin{array}{ll}
\widetilde{A}_{11} & \widetilde{A}_{12} \\
\widetilde{A}_{21} & \widetilde{A}_{22}
\end{array}\right], \quad \widetilde{B}=\left[\begin{array}{l}
\widetilde{B}_{1} \\
\widetilde{B}_{2}
\end{array}\right], \quad C=\left[\widetilde{C}_{1} \widetilde{C}_{2}\right]
$$

we have $\widetilde{A}_{22}=A_{22}, \widetilde{B}_{2}=B_{2}, \widetilde{C}_{2}=C_{2}$ and, for $\hat{S}=\left[I_{\ell} 0_{\ell, r-\ell}\right], \widetilde{A}_{11}=\hat{S} A_{11} \hat{S}^{T}, \widetilde{A}_{12}=\hat{S} A_{12}$, $\widetilde{A}_{21}=A_{21} \hat{S}^{T}, \widetilde{B}_{1}=B_{1} \hat{S}^{T}, \widetilde{C}_{1}=\hat{S} C_{1}$. Consequently, $\widetilde{\mathbf{G}}$ is given by

$$
\begin{aligned}
\widetilde{\mathbf{G}}(s) & =\left(\widetilde{C}_{1}-\widetilde{C}_{2} \widetilde{A}_{22}^{-1} \widetilde{A}_{21}\right)\left(s I_{\ell}-\left(\widetilde{A}_{11}-\widetilde{A}_{12} \widetilde{A}_{22}^{-1} \widetilde{A}_{21}\right)\right)^{-1}\left(\widetilde{B}_{1}-\widetilde{A}_{12} \widetilde{A}_{22}^{-1} \widetilde{B}_{2}\right) \\
& =\left(\left(C_{1}-C_{2} A_{22}^{-1} A_{21}\right) \hat{S}^{T}\right)\left(s I_{\ell}-\hat{S}\left(A_{11}-A_{12} A_{22}^{-1} A_{21}\right) \hat{S}^{T}\right)^{-1}\left(\hat{S}\left(B_{1}-A_{12} A_{22}^{-1} B_{2}\right)\right),
\end{aligned}
$$

Since, furthermore (21) implies that

$$
\begin{aligned}
0= & \left(A_{11}-A_{12} A_{22}^{-1} A_{21}\right) \Gamma+\Gamma\left(A_{11}-A_{12} A_{22}^{-1} A_{21}\right)^{T} \\
& +\left(B_{1}-A_{12} A_{22}^{-1} B_{2}\right)\left(B_{1}-A_{12} A_{22}^{-1} B_{2}\right)^{T}, \\
0= & \left(A_{11}-A_{12} A_{22}^{-1} A_{21}\right)^{T} \Theta+\Theta\left(A_{11}-A_{12} A_{22}^{-1} A_{21}\right) \\
& +\left(C_{1}-C_{2} A_{22}^{-1} A_{21}\right)^{T}\left(C_{1}-C_{2} A_{22}^{-1} A_{21}\right),
\end{aligned}
$$

the inequality (24) is now a direct consequence of the results for the error bound of standard balanced truncation for systems governed by ordinary differential equations (Glover 1984). 


\section{LQG Balanced Realizations}

In this section, we present an LQG balanced truncation model reduction method for the descriptor system (1) which relies on the stabilizing solutions $X$ and $Y$ of the generalized Riccati equations (3) and (4).

\subsection{LQG Gramians and LQG Balancing}

First, we define LQG Gramians and LQG balanced realizations for descriptor systems. This will lead to a new set of invariants for0 descriptor systems, namely, LQG characteristic values.

Definition 4.1: Let $[E, A, B, C, D] \in \Sigma_{n, m, p}$ be strongly stabilizable and strongly detectable, and let $X$ and $Y$ be stabilizing solutions of the generalized Riccati equations (3) and (4), respectively. We call the matrices $E X^{T}$ and $E^{T} Y$, respectively, the $L Q G$ controllability Gramian and the $L Q G$ observability Gramian of $[E, A, B, C, D]$.

Note that, in contrast to stabilizing solutions of the generalized algebraic Riccati equations (3) and (4), the LQG Gramians are unique by Theorem 3.2 (ii). Furthermore they have the following energy interpretation. Consider the available storage, i.e., the maximal energy that can be extracted from system (1), given by

$$
\mathcal{J}_{a s}\left(x_{0}\right)=-\max _{u \in L_{2}\left(\mathbb{R}^{+}\right)^{m}}\left\{-\int_{0}^{\infty}\|u(\tau)\|^{2}+\|y(\tau)\|^{2} d \tau \quad \begin{array}{rl}
E \dot{x} & =A x+B u, x(0)=x_{0}, \\
y & =C x+D u
\end{array}\right\} .
$$

The results in Katayama and Minamino (1992) imply that this functional can be expressed via the LQG observability Gramian as

$$
\mathcal{J}_{a s}\left(x_{0}\right)=x_{0}^{T} E^{T} Y x_{0} .
$$

A similar connection exists between the LQG controllability Gramian and the required supply, i.e., the minimal energy required to steer to a given final state $x_{0} \in \mathbb{R}^{n}$, which is defined by

$$
\mathcal{J}_{r s}\left(x_{0}\right)=\min _{u \in L_{2}\left(\mathbb{R}^{+}\right)^{m}}\left\{\int_{-\infty}^{0}\|u(\tau)\|^{2}+\|y(\tau)\|^{2} d \tau \quad \begin{array}{rl}
E \dot{x}=A x+B u, \quad x(0)=x_{0}, \\
y=C x+D u
\end{array}\right\} .
$$

If system (1) is controllable, we may use a similar argumentation as in the proof of Lemma 3.8 to see that $\operatorname{rank} E=\operatorname{rank}\left(E X^{T}\right)$. Then the required supply is given by

$$
\mathcal{J}_{r s}\left(x_{0}\right)=x_{0}^{T}\left(E X^{T}\right)^{-} x_{0}
$$

where $\left(E X^{T}\right)^{-}$is the symmetric $(1,2)$-pseudoinverse (Campbell and Meyer 1979) of $E X^{T}$.

Definition 4.2: A semi-explicit realization $[E, A, B, C, D] \in \Sigma_{n, m, p}$ of $\mathbf{G} \in \mathbb{R}(s)^{p, m}$ with $r=\operatorname{rank} E$ is called $L Q G$ balanced, if there exists $\Sigma=\operatorname{diag}\left(\sigma_{1}, \ldots, \sigma_{r}\right)$ with $\sigma_{1} \geq \ldots \geq \sigma_{r}>0$, such that the LQG Gramians fulfill

$$
X E^{T}=E^{T} Y=\left[\begin{array}{cc}
\Sigma & 0 \\
0 & 0
\end{array}\right] .
$$

The values $\sigma_{1}, \ldots, \sigma_{r}$ are called $L Q G$ characteristic values of $[E, A, B, C, D]$.

Note that for the semi-explicit LQG balanced realization $[E, A, B, C, D]$, the stabilizing solu- 
tions of the generalized Riccati equations (3) and (4) have the form

$$
X=\left[\begin{array}{cc}
\Sigma & \star \\
0 & \star
\end{array}\right], \quad Y=\left[\begin{array}{cc}
\Sigma & 0 \\
\star & \star
\end{array}\right]
$$

The zero blocks in $X$ and $Y$ evidently occur due to the special structure of $E$ in (11) and the relations $E X^{T}=X E^{T}$ and $E^{T} Y=Y^{T} E$ in (3) and (4).

We now prove the existence of LQG balanced realizations and the invariance property of the LQG characteristic values.

Theorem 4.3: Let $[E, A, B, C, D] \in \Sigma_{n, m, p}$ be a strongly stabilizable and strongly detectable realization of $\mathbf{G} \in \mathbb{R}(s)^{p, m}$. Then the following holds true:

(i) $[E, A, B, C, D]$ is system equivalent to a semi-explicit realization $[\hat{E}, \hat{A}, \hat{B}, \hat{C}, D]$ with the $L Q G$ Gramians

$$
X_{b} \hat{E}^{T}=\left[\begin{array}{ccccc}
\Sigma & 0 & 0 & 0 & 0 \\
0 & \Sigma_{2} & 0 & 0 & 0 \\
0 & 0 & 0 & 0 & 0 \\
0 & 0 & 0 & 0 & 0 \\
0 & 0 & 0 & 0 & 0
\end{array}\right], \quad \hat{E}^{T} \hat{Y}=\left[\begin{array}{ccccc}
\Sigma & 0 & 0 & 0 & 0 \\
0 & 0 & 0 & 0 & 0 \\
0 & 0 & \Sigma_{3} & 0 & 0 \\
0 & 0 & 0 & 0 & 0 \\
0 & 0 & 0 & 0 & 0
\end{array}\right]
$$

for some $\Sigma=\operatorname{diag}\left(\sigma_{1}, \ldots, \sigma_{k}\right)>0, \Sigma_{2}>0$ and $\Sigma_{3}>0$.

(ii) Let $[\hat{E}, \hat{A}, \hat{B}, \hat{C}, D]$ be as in (i), $r=\operatorname{rank} \hat{E}$ and

$$
S_{k}=\left[\begin{array}{ccccc}
I_{k} & 0 & 0 & 0 & 0 \\
0 & 0 & 0 & 0 & I_{n-r}
\end{array}\right] \in \mathbb{R}^{k+n-r, n} .
$$

Then $\left[E_{b}, A_{b}, B_{b}, C_{b}, D\right]=\left[S_{k} \hat{E} S_{k}^{T}, S_{k} \hat{A} S_{k}^{T}, S_{k} \hat{B}, \hat{C} S_{k}^{T}, D\right] \in \Sigma_{k+n-r, m, p}$ is an LQG balanced realization of $\mathbf{G}$.

(iii) The values $\sigma_{1}, \ldots, \sigma_{k}$ are invariants of $\mathbf{G}$, i.e., they do not depend on the particular semiexplicit realization of $\mathbf{G}$.

\section{Proof}

(i) Without loss of generality we can assume that the realization $[E, A, B, C, D]$ is already semi-explicit. The equations $E X^{T}=X E^{T} \geq 0$ and $E^{T} Y=Y^{T} E \geq 0$ then imply the structure

$$
X=\left[\begin{array}{cc}
X_{1} & \star \\
0 & \star
\end{array}\right], \quad Y=\left[\begin{array}{cc}
Y_{1} & 0 \\
\star & \star
\end{array}\right]
$$

where $X_{1}=X_{1}^{T} \geq 0$ and $Y_{1}=Y_{1}^{T} \geq 0$. By Theorem 3.22 of (Zhou et al. 1996), there exists $T_{1} \in \mathrm{Gl}_{r}(\mathbb{R})$ such that

$$
T_{1} X_{1} T_{1}^{T}=\left[\begin{array}{cccc}
\Sigma & 0 & 0 & 0 \\
0 & \Sigma_{2} & 0 & 0 \\
0 & 0 & 0 & 0 \\
0 & 0 & 0 & 0
\end{array}\right], \quad T_{1}^{-T} Y_{1} T_{1}^{-1}=\left[\begin{array}{cccc}
\Sigma & 0 & 0 & 0 \\
0 & 0 & 0 & 0 \\
0 & 0 & \Sigma_{3} & 0 \\
0 & 0 & 0 & 0
\end{array}\right] .
$$

It follows from Lemma 3.5 that for

$$
W=\left[\begin{array}{cc}
T_{1} & 0 \\
0 & I_{n-r}
\end{array}\right], \quad T=\left[\begin{array}{cc}
T_{1}^{-1} & 0 \\
0 & I_{n-r}
\end{array}\right]
$$


the realization $[\hat{E}, \hat{A}, \hat{B}, \hat{C}, D]=[W E T, W A T, W B, C T, D]$ has the desired properties.

(ii) Defining $X_{b}=S_{k} \hat{X} S_{k}^{T}$ and $Y_{b}=S_{k} \hat{Y} S_{k}^{T}$ with $\hat{X}$ and $\hat{Y}$ as in (26), we have

$$
\begin{aligned}
& \hat{C} \hat{X}^{T} S_{k}^{T}=C_{b} X_{b}^{T}, \quad S_{k} \hat{A} \hat{X}^{T} S_{k}^{T}=A_{b} X_{b}^{T}, \\
& \hat{B}^{T} \hat{Y} S_{k}^{T}=B_{b}^{T} X_{b}, \quad S_{k} \hat{A}^{T} \hat{Y} S_{k}^{T}=A_{b}^{T} Y_{b} .
\end{aligned}
$$

These relations imply that $E_{b} X_{b}^{T}$ and $E_{b}^{T} Y_{b}$ are the LQG Gramians of the system $\left[E_{b}, A_{b}, B_{b}, C_{b}, D\right]$. Moreover, by construction of $S_{k}$, we can infer that this realization is LQG balanced. It remains to show that the transfer function of this realization is $\mathbf{G}$.

By Theorem 3.11, we have $\mathbf{G}(s)=\mathbf{N}(s) \mathbf{M}^{-1}(s)$ with $\mathbf{N}(s)$ and $\mathbf{M}(s)$ as in (15). Lemma 3.13 and (28) imply that

$$
\begin{aligned}
{\left[\begin{array}{c}
\mathbf{M}(s) \\
\mathbf{N}(s)
\end{array}\right] } & =\left[\begin{array}{c|c}
s S_{k} \hat{E} S_{k}^{T}-S_{k}\left(\hat{A}-\hat{B} R_{Y}^{-1}\left(\hat{B}^{T} \hat{Y}+D^{T} \hat{C}\right)\right) S_{k}^{T} & S_{k} \hat{B} R_{Y}^{-1 / 2} \\
-R_{Y}^{-1}\left(\hat{B}^{T} \hat{Y}+D^{T} \hat{C}\right) S_{k}^{T} & R_{Y}^{-1 / 2} \\
\left(\hat{C}-D R_{Y}^{-1}\left(\hat{B}^{T} \hat{Y}+D^{T} \hat{C}\right)\right) S_{k}^{T} & D R_{Y}^{-1 / 2}
\end{array}\right], \\
& =\left[\begin{array}{c|c}
s \hat{E}_{b}-\hat{A}_{b}+\hat{B}_{b} R_{Y}^{-1}\left(\hat{B}_{b}^{T} \hat{Y}_{b}+D^{T} \hat{C}_{b}\right) & \hat{B}_{b} R_{Y}^{-1 / 2} \\
\hline-R_{Y}^{-1}\left(\hat{B}_{b}^{T} \hat{Y}_{b}+D^{T} \hat{C}_{b}\right) & R_{Y}^{-1 / 2} \\
\hat{C}_{b}-D R_{Y}^{-1}\left(\hat{B}_{b}^{T} \hat{Y}_{b}+D^{T} \hat{C}_{b}\right) & D R_{Y}^{-1 / 2}
\end{array}\right]
\end{aligned}
$$

Using Theorem 3.11 once again, we obtain that

$$
\mathbf{G}(s)=\mathbf{N}(s) \mathbf{M}^{-1}(s)=D+\hat{C}_{b}\left(s \hat{E}_{b}-\hat{A}_{b}\right)^{-1} \hat{B}_{b},
$$

i.e., $\left[\hat{E}_{b}, \hat{A}_{b}, \hat{B}_{b}, \hat{C}_{b}, D\right] \in \Sigma_{k, m, p}$ is an LQG balanced realization of $\mathbf{G}$.

(iii) This result follows from the fact that the transformations leading one semi-explicit realization into another have the form

$$
W=\left[\begin{array}{cc}
T_{1} & W_{2} \\
0 & W_{3}
\end{array}\right], \quad T=\left[\begin{array}{cc}
T_{1}^{-1} & T_{2} \\
0 & T_{3}
\end{array}\right]
$$

for some $T_{1} \in \mathrm{Gl}_{r}(\mathbb{R}), T_{2}, W_{2} \in \mathbb{R}^{r, n-r}, T_{3}, W_{3} \in \mathrm{Gl}_{n-r}(\mathbb{R})$. Using this structure together with Lemma 3.5, the corresponding results for the standard case (Zhou et al. 1996, p. 75) can be used to obtain the desired result.

\subsection{LQG balanced truncation}

The above construction of an LQG balanced realization may be seen as an errorless model reduction method that removes uncontrollable and unobservable states of $\left[\mathbf{M}^{T}, \mathbf{N}^{T}\right]^{T}$. In the following, we introduce model reduction of descriptor systems by LQG balanced truncation, which is based on further removing the states corresponding to the small LQG characteristic values. Using the energy interpretation for the LQG Gramians, we may conclude that such states have large required supply and small available storage.

Theorem 4.4: Let a realization $[\hat{E}, \hat{A}, \hat{B}, \hat{C}, D] \in \Sigma_{n, m, p}$ of $\mathbf{G} \in \mathbb{R}(s)^{p, m}$ be given with the $L Q G$ Gramians $\hat{E} \hat{X}^{T}$ and $\hat{E}^{T} \hat{Y}$ as in (26) and let the $L Q G$ characteristic values satisfy $\sigma_{1} \geq$ $\ldots \geq \sigma_{\ell}>\sigma_{\ell+1} \geq \ldots \geq \sigma_{k}>0$. Then for

$$
S_{\ell}=\left[\begin{array}{ccccc}
I_{\ell} & 0 & 0 & 0 & 0 \\
0 & 0 & 0 & 0 & I_{n-r}
\end{array}\right] \in \mathbb{R}^{\ell+n-r, n} .
$$


the system $(\widetilde{E}, \widetilde{A}, \widetilde{B}, \widetilde{C}, D)=\left(S_{\ell} \hat{E} S_{\ell}^{T}, S_{\ell} \hat{A} S_{\ell}^{T}, S_{\ell} \hat{B}, \hat{C} S_{\ell}^{T}, D\right) \in \Sigma_{\ell+n-r, m, p}$ is $L Q G$ balanced and its transfer function $\widetilde{\mathbf{G}}(s)=\widetilde{C}(s \widetilde{E}-\widetilde{A})^{-1} \widetilde{B}+D$ satisfies the gap metric estimate

$$
\delta_{g}(\mathbf{G}, \widetilde{\mathbf{G}}) \leq 2 \sum_{j=\ell+1}^{k} \frac{\sigma_{j}}{\sqrt{1+\sigma_{j}^{2}}} .
$$

Proof By using the same argumentation as in the proof of part (ii) of Theorem 4.3, we obtain that the matrices $\widetilde{X}=S_{\ell} \hat{X} S_{\ell}^{T}$ and $\widetilde{Y}=S_{\ell} \hat{Y} S_{\ell}^{T}$ are the LQG Gramians of the realization $(\widetilde{E}, \widetilde{A}, \widetilde{B}, \widetilde{C}, D)$, which is LQG balanced. Since, by Theorem 3.12 , the matrices $P=\left(I+X Y^{T}\right)^{-1} X$ and $Q=Y$ fulfill the generalized Lyapunov equations (16) and (17) and, moreover, the function $f(x)=x^{2} /\left(1+x^{2}\right)$ increases monotonically on $[0, \infty)$, we can apply Lemma 3.13 to obtain that the normalized coprime factorization

$$
\left[\begin{array}{c}
\widetilde{\mathbf{M}}(s) \\
\widetilde{\mathbf{N}}(s)
\end{array}\right]=\left[\begin{array}{c|c}
s \widetilde{E}-\widetilde{A}+\widetilde{B} R_{Y}^{-1}\left(\widetilde{B}^{T} \widetilde{Y}+D^{T} \widetilde{C}\right) & \widetilde{B} R_{Y}^{-1 / 2} \\
\hline-R_{Y}^{-1}\left(\widetilde{B}^{T} \widetilde{Y}+D^{T} \widetilde{C}\right) & R_{Y}^{-1 / 2} \\
\widetilde{C}-D R_{Y}^{-1}\left(\widetilde{B}^{T} \widetilde{Y}+D^{T} \widetilde{C}\right) & D R_{Y}^{-1 / 2}
\end{array}\right]
$$

of $\widetilde{\mathbf{G}}$ satisfies

$$
\left\|\left[\begin{array}{c}
\widetilde{\mathbf{M}}-\mathbf{M} \\
\widetilde{\mathbf{N}}-\mathbf{N}
\end{array}\right]\right\|_{\infty} \leq 2 \sum_{j=\ell+1}^{k} \frac{\sigma_{j}}{\sqrt{1+\sigma_{j}^{2}}}
$$

Then the gap metric error bound (29) immediately follows from Theorem 2.4.

Remark 1: It is important to note that it is not required to compute stabilizing solutions which have the additional property that $I+X Y^{T}$ is invertible. This is because, in the proof of Theorem 4.3, only the LQG Gramians $E X^{T} \geq 0$ and $E^{T} Y \geq 0$ (and not the full stabilizing solutions $X$ and $Y$ ) were used to construct the LQG balanced realization. Roughly speaking, this means that the $\star$-blocks in (27) do not play a role for the construction of an LQG balanced realization. The existence of stabilizing solutions $X$ and $Y$ with invertible $I+X Y^{T}$ is only used for the solution structure of the generalized Lyapunov equations (16) and (17) and, hence, for the proof of the gap metric error bound (29).

Remark 2: For an accordant block partitioning

$$
\hat{E}=\left[\begin{array}{ccc}
I_{\ell} & 0 & 0 \\
0 & I_{r-\ell} & 0 \\
0 & 0 & 0
\end{array}\right], \quad \hat{A}=\left[\begin{array}{lll}
A_{11} & A_{12} & A_{13} \\
A_{21} & A_{22} & A_{23} \\
A_{31} & A_{32} & A_{33}
\end{array}\right], \quad \hat{B}=\left[\begin{array}{l}
B_{1} \\
B_{2} \\
B_{3}
\end{array}\right], \quad \hat{C}=\left[C_{1}, C_{2}, C_{3}\right]
$$

of a realization $[\hat{E}, \hat{A}, \hat{B}, \hat{C}, D] \in \Sigma_{n, m, p}$ of $\mathbf{G} \in \mathbb{R}(s)^{p, m}$ with the LQG Gramians $\hat{E} \hat{X}^{T}$ and $\hat{E}^{T} \hat{Y}$ as in (26) and the LQG characteristic values $\sigma_{1} \geq \ldots \geq \sigma_{\ell}>\sigma_{\ell+1} \geq \ldots, \sigma_{k}>0$, the reduced-order model by LQG balanced truncation is given by $[\widetilde{E}, \widetilde{A}, \widetilde{B}, \widetilde{C}, D] \in \Sigma_{\ell+n-r, m, p}$ with

$$
\widetilde{E}=\left[\begin{array}{rr}
I_{\ell} & 0 \\
0 & 0
\end{array}\right], \quad \widetilde{A}=\left[\begin{array}{ll}
A_{11} & A_{13} \\
A_{31} & A_{33}
\end{array}\right], \quad \widetilde{B}=\left[\begin{array}{l}
B_{1} \\
B_{3}
\end{array}\right], \quad \widetilde{C}=\left[C_{1}, C_{3}\right] .
$$

LQG balanced truncation, therefore, only eliminates differential variables while letting the algebraic constraints unchanged. It is possible to reduce the algebraic part by eliminating the uncontrollable and unobservable components (Varga 1990). 
In the time domain, the gap metric error bound (29) has the following interpretation. For normalized coprime factorizations

$$
\left[\begin{array}{l}
\mathbf{M}(s) \\
\mathbf{N}(s)
\end{array}\right]=\left[\begin{array}{c|c}
s E_{\mathrm{rcf}}-A_{\mathrm{rcf}} & B_{\mathrm{rcf}} \\
\hline C_{\mathrm{rcf}} & D_{\mathrm{rcf}}
\end{array}\right], \quad\left[\begin{array}{c}
\widetilde{\mathbf{M}}(s) \\
\widetilde{\mathbf{N}}(s)
\end{array}\right]=\left[\begin{array}{c|c}
s \widetilde{E}_{\mathrm{rcf}}-\widetilde{A}_{\mathrm{rcf}} & \widetilde{B}_{\mathrm{rcf}} \\
\hline \widetilde{C}_{\mathrm{rcf}} & D_{\mathrm{rcf}}
\end{array}\right],
$$

the set of input-output pairs of the original and reduced-order models can be parameterized as

$$
\begin{aligned}
E_{\mathrm{rcf}} \dot{x}(t) & =A_{\mathrm{rcf}} x(t)+B_{\mathrm{rcf}} v(t), & \widetilde{E}_{\mathrm{rcf}} \dot{\vec{x}}(t) & =\widetilde{A}_{\mathrm{rcf}} \widetilde{x}(t)+\widetilde{B}_{\mathrm{rcf}} v(t), \\
{\left[\begin{array}{l}
u(t) \\
y(t)
\end{array}\right] } & =C_{\mathrm{rcf}} x(t)+D_{\mathrm{rcf}} v(t), & {\left[\begin{array}{l}
\widetilde{u}(t) \\
\widetilde{y}(t)
\end{array}\right] } & =\widetilde{C}_{\mathrm{rcf}} \widetilde{x}(t)+D_{\mathrm{rcf}} v(t),
\end{aligned}
$$

where $v: \mathbb{R}^{+} \rightarrow \mathbb{R}^{m}$ is the so-called driving variable (Weiland 1994). The property of the coprime factorization being normalized, means that both systems in (31) are inner (Zhou et al. 1996, pp. 357). This implies that the $L_{2}$-norms of the driving variable and the input-output pairs coincide, i.e.,

$$
\left\|\left[\begin{array}{l}
u \\
y
\end{array}\right]\right\|_{\mathcal{L}_{2}}=\left\|\left[\begin{array}{c}
\widetilde{u} \\
\widetilde{y}
\end{array}\right]\right\|_{\mathcal{L}_{2}}=\|v\|_{\mathcal{L}_{2}}
$$

Hence, the $\mathcal{H}_{\infty}$-error bound (30) for the coprime factor system gives rise to the following behavioral error bound.

Lemma 4.5: Let

$$
\left[\begin{array}{l}
u \\
y
\end{array}\right] \in \mathcal{L}_{2}^{m+p}\left(\mathbb{R}^{+}\right)
$$

be an input-output pair of the descriptor system (1) with $x(0)=0$. Then there exists an inputoutput pair

$$
\left[\begin{array}{l}
\widetilde{u} \\
\widetilde{y}
\end{array}\right] \in \mathcal{L}_{2}^{m+p}\left(\mathbb{R}^{+}\right)
$$

of the descriptor system (2) with $\widetilde{x}(0)=0$ such that

$$
\left\|\left[\begin{array}{c}
u-\widetilde{u} \\
y-\widetilde{y}
\end{array}\right]\right\|_{\mathcal{L}_{2}} \leq 2 \sum_{j=\ell+1}^{k} \frac{\sigma_{j}}{\sqrt{1+\sigma_{j}^{2}}}\left\|\left[\begin{array}{l}
u \\
y
\end{array}\right]\right\|_{\mathcal{L}_{2}} .
$$

We now aim to give a constructive approach to LQG balanced truncation. It is obvious that, for the construction of a reduced-order model, there is no need to compute first a full LQG balanced realization as in part (i) of Theorem 4.3, and then truncate the states corresponding to the small LQG characteristic values. Instead, we can combine these two steps similarly to the classical balanced truncation (Tombs and Postlethwaite 1987).

By Theorem 3.2 (iii) and (iv), the LQG Gramians have the property that $X E^{T} \geq 0$ and $E^{T} Y \geq 0$. Then there exist factorizations

$$
E X^{T}=E R R^{T} E^{T}, \quad E^{T} Y=E^{T} L^{T} L E
$$


for some full row rank matrices $L$ and $R^{T}$. Computing a singular value decomposition

$$
L E R=\left[U_{1}, U_{2}\right]\left[\begin{array}{cc}
\Sigma_{1} & 0 \\
0 & \Sigma_{2}
\end{array}\right]\left[V_{1}, V_{2}\right]^{T}
$$

where $\left[U_{1}, U_{2}\right]$ and $\left[V_{1}, V_{2}\right]$ are orthogonal, $\Sigma_{1}=\operatorname{diag}\left(\sigma_{1}, \ldots, \sigma_{\ell}\right)$ and $\Sigma_{2}=\operatorname{diag}\left(\sigma_{\ell+1}, \ldots, \sigma_{k}\right)$, we can determine the projection matrices

$$
W_{\ell}=\left[L^{T} U_{1} \Sigma_{1}^{-1 / 2}, Z_{l}\right], \quad T_{\ell}=\left[R V_{1} \Sigma_{1}^{-1 / 2}, Z_{r}\right],
$$

where $Z_{r}, Z_{l} \in \mathbb{R}^{n, n-r}$ are full column rank matrices satisfying $\operatorname{im} Z_{r}=\operatorname{ker} E, \operatorname{im} Z_{l}=\operatorname{ker} E^{T}$. Then the reduced-order system $[\widetilde{E}, \widetilde{A}, \widetilde{B}, \widetilde{C}, D]=\left[W_{\ell}^{T} E T_{\ell}, W_{\ell}^{T} A T_{\ell}, W_{\ell}^{T} B, C T_{\ell}, D\right]$ is semiexplicit and LQG balanced. Indeed, we have

$$
\begin{aligned}
\widetilde{E}=W_{\ell}^{T} E T_{\ell} & =\left[\begin{array}{cc}
\Sigma_{1}^{-1 / 2} U_{1}^{T} L E R V_{1} \Sigma_{1}^{-1 / 2} & \Sigma_{1}^{-1 / 2} U_{1}^{T} L E Z_{r} \\
Z_{l}^{T} E R V_{1} \Sigma_{1}^{-1 / 2} & Z_{\ell}^{T} E Z_{r}
\end{array}\right]=\left[\begin{array}{cc}
I & 0 \\
0 & 0
\end{array}\right], \\
W_{\ell}^{T} X E^{T} W_{\ell} & =\left[\begin{array}{cc}
\Sigma_{1}^{-1 / 2} U_{1}^{T} L X E^{T} L^{T} U_{1} \Sigma_{1}^{-1 / 2} & \Sigma_{1}^{-1 / 2} U_{1}^{T} L X E^{T} Z_{l} \\
Z_{l}^{T} X E^{T} L^{T} U_{1} \Sigma_{1}^{-1 / 2} & Z_{l}^{T} X E^{T} Z_{l}
\end{array}\right]=\left[\begin{array}{cc}
\Sigma & 0 \\
0 & 0
\end{array}\right], \\
T_{\ell}^{T} E^{T} Y T_{\ell} & =\left[\begin{array}{cc}
\Sigma_{1}^{-1 / 2} V_{1}^{T} R^{T} E^{T} Y R V_{1} \Sigma_{1}^{-1 / 2} & \Sigma_{1}^{-1 / 2} V_{1}^{T} R^{T} E^{T} Y Z_{r} \\
Z_{r}^{T} E^{T} Y R V_{1} \Sigma_{1}^{-1 / 2} & Z_{r}^{T} E Y^{T} Z_{r}
\end{array}\right]=\left[\begin{array}{cc}
\Sigma & 0 \\
0 & 0
\end{array}\right] .
\end{aligned}
$$

We summarize the LQG balanced truncation method for descriptor systems in the following algorithm.

Algorithm 1: LQG balanced truncation for descriptor systems.

Given a realization $[E, A, B, C, D]$ of a strongly stabilizable and strongly detectable descriptor system (1) with $r=\operatorname{rank} E$, compute an LQG balanced realization $[\widetilde{E}, \widetilde{A}, \widetilde{B}, \widetilde{C}, \widetilde{D}]$ of a reducedorder model (2).

(1) Compute full rank matrices $Z_{r}, Z_{l} \in \mathbb{R}^{n, n-r}$ such that im $Z_{r}=\operatorname{ker} E$ and $\operatorname{im} Z_{l}=\operatorname{ker} E^{T}$.

(2) Compute the full rank factors $L \in \mathbb{R}^{r_{c}, n}$ and $R \in \mathbb{R}^{n, r_{o}}$ such that $E X^{T}=E R R^{T} E^{T}$ and $E^{T} Y=E^{T} L^{T} L E$ by solving the generalized algebraic Riccati equations (3) and (4).

(3) Compute a singular value decomposition (33).

(4) Compute the reduced-order system

$$
\widetilde{E}=W_{\ell}^{T} E T_{\ell}, \quad \widetilde{A}=W_{\ell}^{T} A T_{\ell}, \quad \widetilde{B}=W_{\ell}^{T} B, \quad \widetilde{C}=C T_{\ell}, \quad \widetilde{D}=D
$$

with the projection matrices $W_{\ell}$ and $T_{\ell}$ as in (34).

\section{$5 \quad$ Examples}

In this section we present two numerical examples to demonstrate the feasibility of the proposed LQG balanced truncation model reduction method for descriptor systems. The computations were done on IBM RS 6000 44P Model 270 with machine precision $\varepsilon=2.22 \times 10^{16}$ using MATLAB 7.9.0.

To present the accuracy of the reduced order models, we plot the norm of difference between the normalized coprime factorizations of the reduced order model and those of the full order 


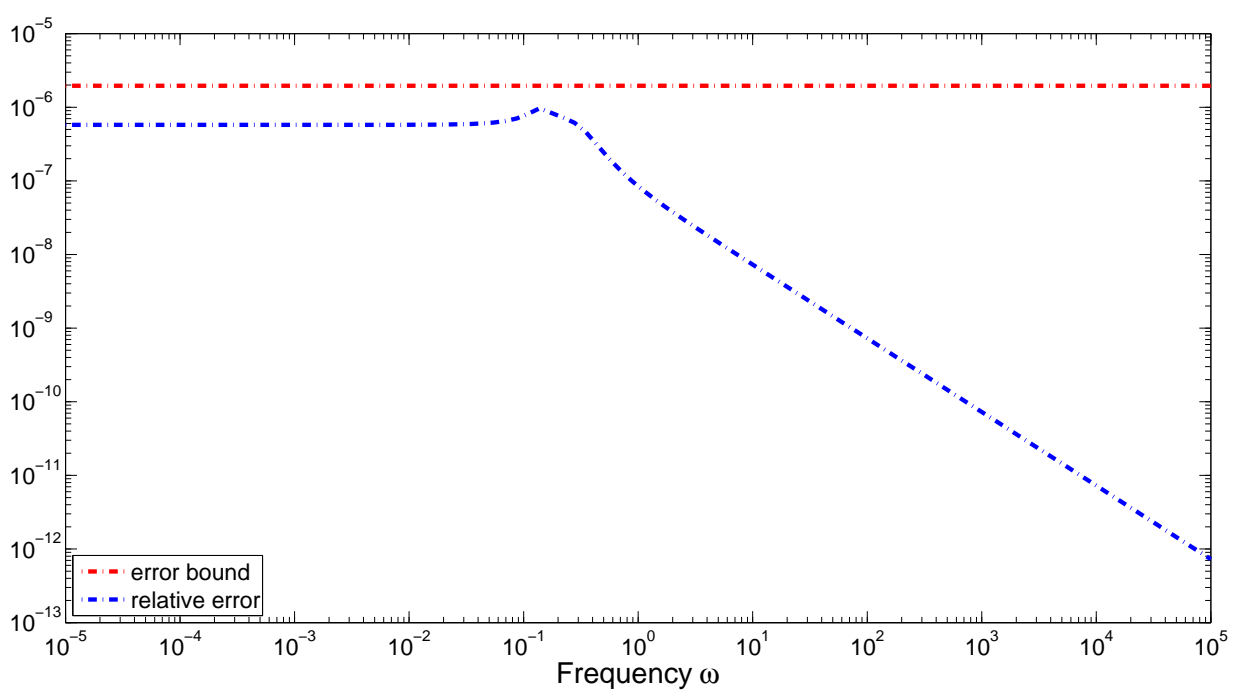

Figure 1. Coprime factor error plot and error bound for Example 5.1

models. That is, we plot

$$
\left\|\left[\begin{array}{l}
\mathbf{M}(i \omega)-\widetilde{\mathbf{M}}(i \omega) \\
\mathbf{N}(i \omega)-\widetilde{\mathbf{N}}(i \omega)
\end{array}\right]\right\|
$$

for a frequency range $\omega \in\left[10^{-5}, 10^{5}\right]$. Note that, since the coprime factorizations are normalized, i.e.

$$
\left\|\left[\begin{array}{l}
\mathbf{M}(i \omega) \\
\mathbf{N}(i \omega)
\end{array}\right]\right\|=1 \quad \text { for all } \omega \in \mathbb{R}
$$

the computed error is the equal to the relative error. Furthermore we present the error bound, computed by (29).

Example 5.1 As a first example we consider a mechanical system with holonomic constraints. Such a system can be described by model equations in the form (1), where the system matrices have the following blockstructure

$$
E=\left[\begin{array}{lll}
I & & \\
& M & \\
& & 0
\end{array}\right], \quad A=\left[\begin{array}{ccc}
0 & I & 0 \\
K & D & -G^{T} \\
G & 0 & 0
\end{array}\right], \quad B=\left[\begin{array}{c}
0 \\
B_{2} \\
0
\end{array}\right], \quad C=\left[C_{1}, C_{2}, C_{3}\right]
$$

Here $M, K, D$ are the mass, stiffness and damping matrices respectivel,and y $G$ is the full row rank constraint matrix. This system is stable and has index 3 . The system dimensions are $n=1001, m=2$ and $p=3$. We approximate the resulted unstable system by a reduced order model of dimension $l=20$. To show that the presented model reduction approach works also for unstable models, we destabilize this system by a proportional state feedback. Figure 1 displays the relative error (35) and the computed error bound (29)

Example 5.2 We consider now the instationary Stokes equation describing the flow of an incompressible fluid in a domain (Schmidt 2007, Section 3.7.1). Discretization of this equation by a finite volume method on a uniform staggered grid leads to the descriptor system (1) of 


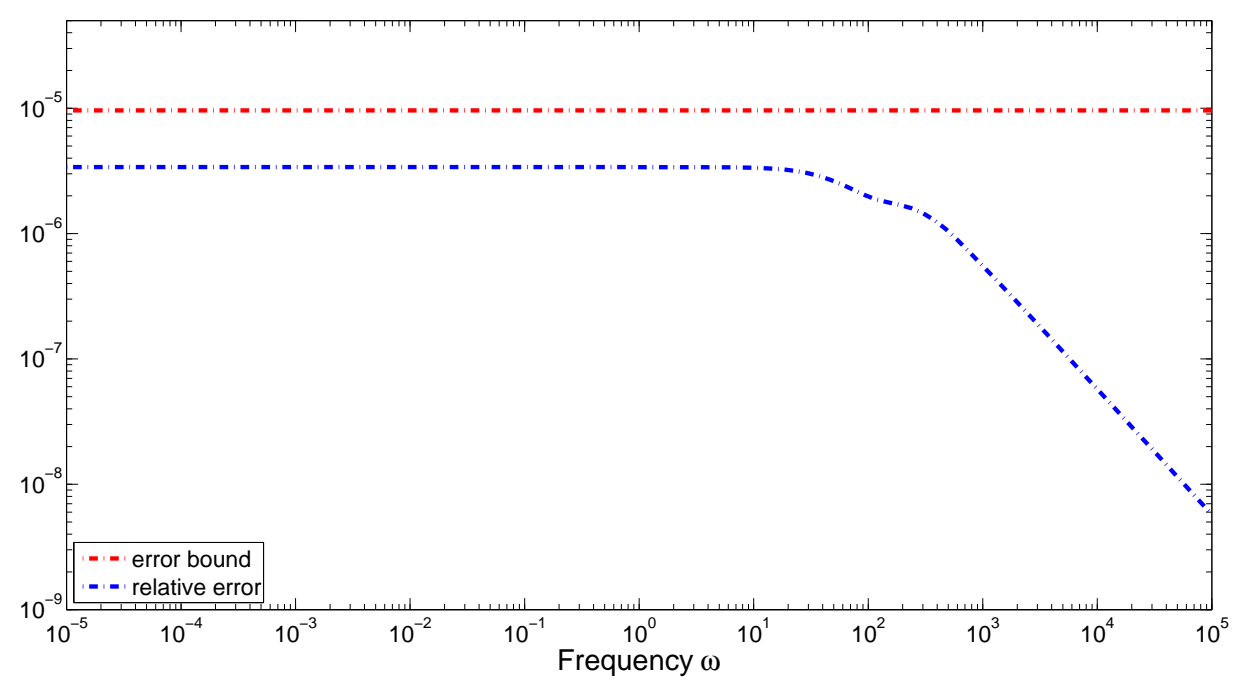

Figure 2. Relative error plot and error bound for Example 5.2

index 2 with the matrix coefficients

$$
E=\left[\begin{array}{ll}
I & 0 \\
0 & 0
\end{array}\right], \quad A=\left[\begin{array}{cc}
A_{11} & A_{12} \\
A_{12}^{T} & 0
\end{array}\right], \quad B=\left[\begin{array}{c}
B_{1} \\
0
\end{array}\right], \quad C=\left[C_{1}, C_{2}\right]
$$

In our experiments, the system dimensions are $n=2640, m=3$ and $p=2$. Since the system is neither strongly stabilizable nor strongly detectable we first remove the states that are not impulse controllable and not impulse observable using the staircase-based algorithm form (Varga 1990). As a result we obtained a system of state space dimension 869. This system was then approximated by a reduced model of dimension $\ell=38$. The relative error (35) and the corresponding error bound are presented in Figure 2.

\section{Conclusion}

In this paper we have presented the linear-quadratic Gaussian (LQG) balanced truncation method for linear time-invariant differential-algebraic systems. This method is also applicable to unstable systems, provides an error bound in terms of the gap metric and is based on balancing the solutions of certain generalized algebraic Riccati equations. We have also presented systems theoretic conditions on the solvability of these equations.

\section{Acknowledgments}

This work is supported by the Research Network FROPT - Model reduction based optimal control methods for field-flow-fractionation funded by the German Federal Ministry of Education and Science (BMBF), grant 03MS612G.

\section{References}

Bunse-Gerstner, A., Byers, R., Mehrmann, V., and Nichols, N. (1999), "Feedback design for regularizing descriptor systems," Linear Algebra Appl., 299, 119-151.

Campbell, S., and Meyer, C., Generalized Inverses of Linear Transformations, New York: Dover Publications (1979). 
Curtain, R. (2003), "On model reduction for control design for distributed parameter systems," in Research Directions in Distributed Parameter Systems eds. R. Smith and M. Demetriou, Philadelphia, PA: SIAM, pp. 95-121.

Dai, L., Singular Control Systems, Vol. 118 of Lecture Notes in Control and Information Sciences, Berlin, Heidelberg: Springer-Verlag (1989).

Gantmacher, F., Theory of Matrices, Vol. 2, New York: Chelsea (1959).

Georgiou, T., and Smith, M. (1990), "Optimal robustness in the gap metric," IEEE Trans. Automat. Control, 35, 673-686.

Glover, K. (1984), "All optimal Hankel-norm approximations of linear multivariable systems and their $L^{\infty}$-error bounds," Internat. J. Control, 39, 1115-1193.

Gugercin, S., and Antoulas, A. (2004), "A survey of model reduction by balanced truncation and some new results," Internat. J. Control, 77, 748-766.

Jonckheere, E., and Silverman, L. (1983), "A new set of invariants for linear systems with application to reduced order compensator," IEEE Trans. Automat. Control, 28, 953-964.

Katayama, T., and Minamino, K. (1992), "Linear quadratic regulator and spectral factorization for continous-time descriptor systems," in Proceedings of the 31st IEEE Conference on Decision and Control, pp. $967-972$ vol.1.

Kawamoto, A., Takaba, K., and Katayama, T. (1999), "On the generalized algebraic Riccati equation for continuous-time descriptor systems," Linear Algebra Appl., 296, 1-14.

Lancaster, P., and Rodman, L., Algebraic Riccati equations, Oxford: Clarendon Press (1995).

Liu, W., Sreeram, V., Teo, K., and Xie, B. (1997), "Normalized coprime factorization for singular systems," in Proceedings of the American Control Conference, Albuquerque, NM, June 4-6, pp. $2125-2126$.

Moore, B. (1981), "Principal component analysis in linear systems: controllability, observability, and model reduction," IEEE Trans. Automat. Control, 26, 17-32.

Schmidt, M. (2007), "Systematic Discretization of Input/Output Maps and other Contributions to the Control of Distributed Parameter Systems," Technische Universität Berlin.

Sefton, J., and Ober, R. (1993), "On the gap metric and coprime factor perturbations," Automatica, 29, 723-734.

Stykel, T. (2004), "Gramian-based model reduction for descriptor systems," Math. Control Signals Systems, 16, 297-319.

Takaba, K., Morihira, N., and Katayama, T. (1994), "A generalized Lyapunov theorem for descriptor system," Systems Control Lett., 24, 49-51.

Thompson, R. (1976), "The characteristic polynomial of a principal subpencil of a Hermitian matrix pencil," Linear Algebra Appl., 14, 135-177.

Tombs, M., and Postlethwaite, I. (1987), "Truncated balanced realization of a stable non-minimal state-space system," Internat. J. Control, 46, 1319-1330.

Varga, A. (1990), "Computation of irreducible generalized state-space realizations," Kybernetika (Prague), 26, 89-106.

Vidyasagar, V. (2001), "The graph metric for unstable plants and robustness estimates for feedback stability," IEEE Trans. Automat. Control, 29, 403-418.

Weiland, S. (1994), "A behavioral approach to balanced representations of dynamical systems," Linear Algebra Appl., 205-206, 1227-1252.

Xin, X. (2008), "Strong solutions and maximal solutions of generalized algebraic Riccati equations," in Proceedings of the 47th IEEE Conference on Decision and Control, Cancun, Mexico, December 9-11, IEEE, pp. 528-533.

Yang, D., Zhang, Q., and Zhang, G. (2001), " $H_{2}$ Algebraic Riccati equation for descriptor systems," in Proceedings of American Control Conference, Arlington, VA, June 25-27, pp. $2937-2942$.

Zhang, X., Zhong, G.P., and Tan, C. (2009), "Existence and representations of stabilizing solutions to generalized algebraic Riccati equations," in Proceedings of the 48th IEEE Conference on Decision and Control, Shanghai, China, December 16-18, pp. 5923-5928. 
Zhou, K., Doyle, J., and Glover, K., Robust and Optimal Control, Princeton: Prentice-Hall (1996). 